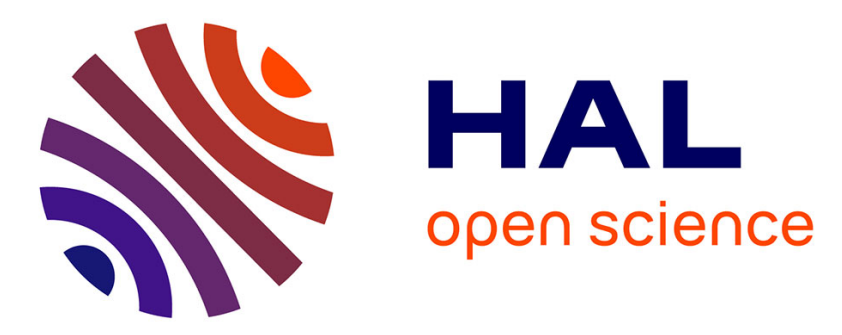

\title{
Donor-acceptor-donor structured thioxanthone derivatives as visible photoinitiators
}

\author{
Alexandre Mau, Thi Huong Le, Céline Dietlin, Thanh-Tuân Bui, Bernadette \\ Graff, Frédéric Dumur, Fabrice Goubard, Jacques Lalevee
}

\section{- To cite this version:}

Alexandre Mau, Thi Huong Le, Céline Dietlin, Thanh-Tuân Bui, Bernadette Graff, et al.. Donoracceptor-donor structured thioxanthone derivatives as visible photoinitiators. Polymer Chemistry, 2020, 11 (45), pp.7221-7234. 10.1039/D0PY01244K . hal-03029059

\section{HAL Id: hal-03029059 \\ https://hal.science/hal-03029059}

Submitted on 27 Nov 2020

HAL is a multi-disciplinary open access archive for the deposit and dissemination of scientific research documents, whether they are published or not. The documents may come from teaching and research institutions in France or abroad, or from public or private research centers.
L'archive ouverte pluridisciplinaire HAL, est destinée au dépôt et à la diffusion de documents scientifiques de niveau recherche, publiés ou non, émanant des établissements d'enseignement et de recherche français ou étrangers, des laboratoires publics ou privés. 


\title{
Donor-acceptor-donor structured thioxanthone derivatives as visible photoinitiators
}

\author{
Alexandre Mau ${ }^{\mathrm{ab}}$, Thi Huong Le ${ }^{\mathrm{c}}$, Céline Dietlin ${ }^{\mathrm{a}}$, Thanh-Tuân Bui *c, \\ Bernadette Graff ${ }^{\mathrm{a}}{ }^{\mathrm{b}}$, Frédéric Dumur ${ }^{\mathrm{d}}$, Fabrice Goubard ${ }^{\mathrm{c}}$, and Jacques Lalevee*a \\ ${ }^{a}$ Université de Haute-Alsace, CNRS, IS2M UMR 7361, F-68100 Mulhouse, France \\ ${ }^{\mathrm{b}}$ Université de Strasbourg, France \\ ${ }^{c}$ CY Cergy Paris Université, LPPI, F-95000 Cergy, France \\ ${ }^{d}$ Aix Marseille Univ, CNRS, ICR, UMR 7273, F-13397 Marseille, France \\ * Corresponding author: tbui @ cyu.fr (T.-T. B.); jacques.lalevee@uha.fr (J. L.)
}

\begin{abstract}
Three thioxanthone derivatives differing by their peripheral groups have been investigated as visible light photoinitiators of polymerisation. Their reactivity and efficiency have been compared with that of a commercial type II photoinitiator (2-isopropylthioxanthone - ITX) in the case of free radical polymerisation, cationic polymerisation and interpenetrated polymer networks synthesis for $25 \mu \mathrm{m}$ or $1.4 \mathrm{~mm}$ thick samples under a $405 \mathrm{~nm}$ LED irradiation. They are incorporated into either, a two-component system with an iodonium salt or an amine, or a three-component system combining an iodonium salt and an amine. Using absorption and fluorescence spectroscopies, laser flash photolysis and molecular modelling, optical properties, excited state energies and lifetimes of these thioxanthone derivatives have been determined allowing a better understanding of the associated chemical mechanisms. Interestingly, the main reaction pathway for one of these thioxanthone derivatives in the photoinitiating systems was determined as involving its singlet state $\mathrm{S}_{1}$ whereas ITX and thioxanthones are known to react from their triplet excited state $\mathrm{T}_{1}$. The high efficiency of the new initiating systems was found as being worthwhile for laser write applications @ $405 \mathrm{~nm}$ but also for high migration stability.
\end{abstract}

\section{Introduction}

Polymerisation initiated with visible light still remains an important challenge even after four to five decades of development. Indeed, not only the energy consumption is reduced compared to that used for UV-initiated polymerisation and the light penetration capability higher, but this process is also much safer which explained the development of various applications in dentistry ${ }^{1}$, coatings ${ }^{2}$ or $3 \mathrm{D}$-printing ${ }^{3}$. Therefore, different photoinitiators and photoinitiating systems have been developed to initiate either free radical polymerisations or cationic polymerisations under mild reaction conditions (room temperature, visible light, under air). One strategy to achieve this goal consists in designing new photoinitiating systems by modifying the chemical structure of well-known photoinitiators absorbing in near UV such as coumarins ${ }^{4}$ and thioxanthones 5678 .

Over the past decades, various studies have been conducted on designing thioxanthone-based photoinitiating systems and three main approaches could be inferred: the design of polymeric

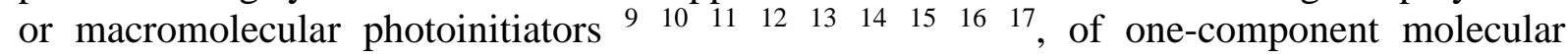
photoinitiators ${ }^{7181920}$ and of multi-component molecular photoinitiating systems ${ }^{21} 222324$. For example, Dadashi-Silab et al. ${ }^{14}$ proposed microporous polymers, where thioxanthone moieties were integrated into the polymer backbones as type II photoinitiators able to initiate both the free radical and cationic polymerisations. Recently, Wu et al. ${ }^{9}$ and Valandro et al. ${ }^{11}$ developed 
interesting polymeric photoinitiators with thioxanthone moieties on the side chain of respectively silicone and chitosan-based polymers which allowed a decrease of the photoinitiator migration due to the drastic increase of the photoinitiator molecular weight. Wu et al. ${ }^{7}$ also designed thioxanthone based one-component photoinitiators for the free radical polymerisation of various multifunctional acrylates under xenon lamp. The design of these onecomponent photoinitiators was based on the functionalisation of thioxanthone with a coinitiator moiety (hydrogen donor). Wu et al. ${ }^{21}$ developed high performance visible light multicomponent photoinitiating systems. Based on the reactivity of a substituted isopropylthioxanthone, an iodonium salt and a co-initiator, these multi-component photoinitiating systems could efficiently initiate free radical and cationic polymerisations under $405 \mathrm{~nm}$ LED irradiation of $40 \mu \mathrm{m}$ thick samples.

In this context, a compound with a donor-acceptor-donor structure (D-A-D structure) where the thioxanthone moiety acts as the acceptor can be an interesting candidate for a photoinitiating system due to its improved light absorption properties associated with the presence of the intramolecular charge transfer. Precisely, compared to the parent thioxanthone, D-A-D triads are expected to exhibit both a red-shifted absorption combined with an enhancement of the molar extinction coefficient. In the literature, such triads have already been designed with carbazole moieties as electron donors ${ }^{25}$. These structures were notably designed as fluorescent emitters exhibiting thermally activated delayed fluorescence (TADF) properties for the elaboration of high-performance organic light emitting diodes (OLEDs).

In this work, three D-A-D structured thioxanthone derivatives, presented in Figure 1, with different peripheral electron donors were investigated as photoinitiators. The free radical polymerisation of acrylates and the cationic polymerisation of epoxides with two-component initiating systems were examined upon visible light irradiation. Finally, the synthesis of interpenetrated polymer networks (IPNs) using three-component initiating systems was also examined. To evidence the interest of these new structures, the reactivity, efficiency and optical properties of the new photoinitiators were compared to that of a benchmark thioxanthone photoinitiator i.e. 2-isopropylthioxanthone ITX.
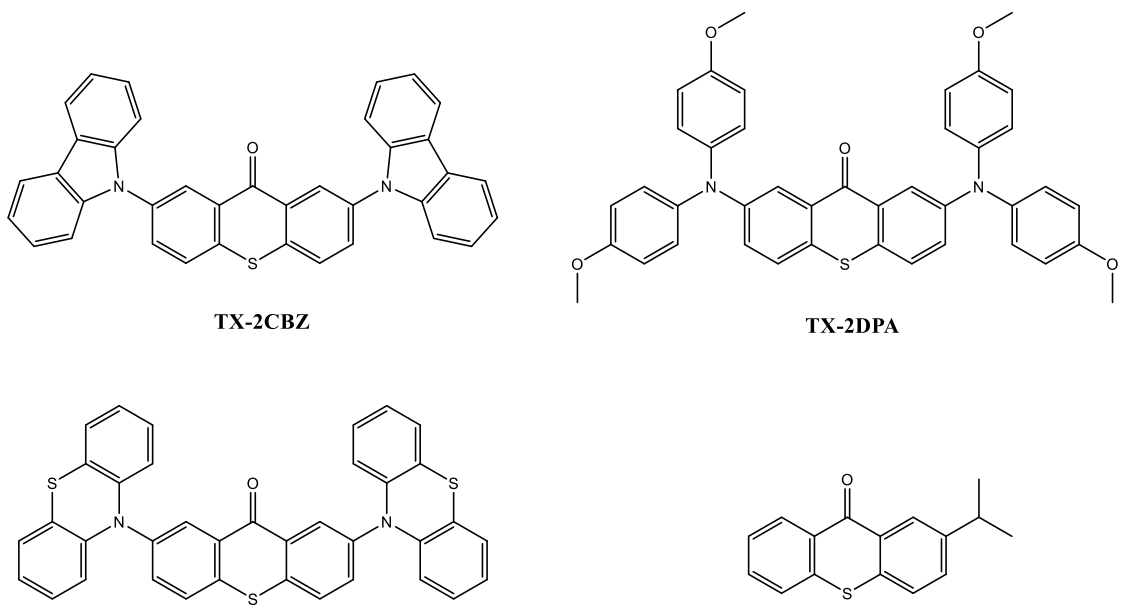

TX-2PTZ

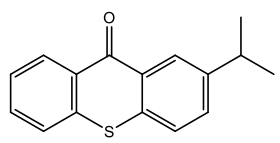

ITX

Figure 1: Chemical structures of the investigated thioxanthones and the benchmark photoinitiator (ITX) 


\section{Experimental section 2.1. Chemical Compounds}

Bis-(4-tert-butylphenyl)iodonium hexafluorophosphate (Iod; SpeedCure 938), ethyl 4(dimethylamino)benzoate (EDB; SpeedCure EDB) and 2-isopropylthioxanthone (ITX; SpeedCure 2-ITX) were obtained from Lambson Ltd (UK). Trimethylolpropane triacrylate (TMPTA) and (3,4-epoxycyclohexane)methyl-3,4-epoxycyclohexylcarboxylate (EPOX; Uvacure 1500) were obtained from Allnex and used as benchmark monomers for radical and cationic photopolymerisation, respectively (Figure 2). Dichloromethane (DCM, purity $\geq 99 \%$ ) used as solvent, quinine hemisulphate salt monohydrate (BioReagent, suitable for fluorescence, purity 99.0-101.0\%), 1-methylnaphthalene (purity 95\%), benzophenone (ReagentPlus, purity $99 \%$ ) and colloidal silica suspension (LUDOX ${ }^{\circledR}$ AS 30, 30wt\% suspension in $\mathrm{H}_{2} \mathrm{O}$ ) were purchased from Sigma-Aldrich.<smiles>CCOC(=O)c1ccc(N(C)C)cc1</smiles>

EDB

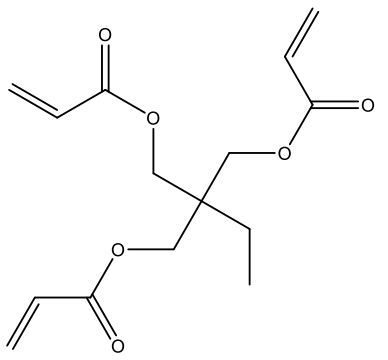

TMPTA<smiles>CC(C)(C)c1ccc(Oc2ccc(C(C)(C)C)cc2)cc1</smiles>

Iod<smiles>O=C(OCC1CCC2OC2C1)C1CCC2OC2C1</smiles>

EPOX

Figure 2: Chemical structures of additives and monomers

\subsection{Synthesis of investigated thioxanthone derivatives:}

The investigated thioxanthones were synthesised and characterised according to the following protocol. All the chemicals and solvents were purchased from chemical companies and used as received, unless otherwise mentioned. Purification of products was performed by column chromatography on silica gel from Merck with a grain size of 0.04-0.063 mm (flash silica gel, Geduran Si 60) eluting with analytically pure solvents. NMR $\left({ }^{1} \mathrm{H}\right.$ and $\left.{ }^{13} \mathrm{C}\right)$ spectra were recorded on a Bruker DPX-250 FT- NMR spectrometer. Chemical shifts are given in ppm using the residual solvent signal as internal reference. Mass spectroscopy was performed by the Spectropole of Aix-Marseille University. Electron spray ionization (ESI) mass spectral analyses were recorded with a 3200 QTRAP (Applied Biosystems SCIEX) mass spectrometer. The HRMS mass spectral analysis was performed with a QStar Elite (Applied Biosystems SCIEX) mass spectrometer. All compounds were prepared from 2,7-dibromo- $9 \mathrm{H}$-thioxanthen9-one, a known compound ${ }^{26}$. The syntheses of TX-2DPA and TX-2PTZ have been described elsewhere in a separate publication. The synthesis of TX-2CBZ, which was already known in literature ${ }^{25}$, was performed according to the following protocol. 
2,7-di(9H-carbazol-9-yl)-9H-thioxanthen-9-one (TX-2CBZ): In a dried Schlenk flask, the mixture of 2,7-dibromo-9H-thioxanthen-9-one (200 mg, $1.0 \mathrm{eq})$ and carbazole $96 \%$ (300 mg, $3.0 \mathrm{eq})$, CuI (20 mg, $0.2 \mathrm{eq})$, sodium carbonate ( $0.3 \mathrm{~g}, 5.0 \mathrm{eq})$ and 18 -crown-6 (30 mg, $0.2 \mathrm{eq})$ were added at once. Then, $15 \mathrm{~mL}$ of nitrobenzene was supplied. The reaction mixture was stirred under argon atmosphere while the temperature was slowly raised up to $160^{\circ} \mathrm{C}$ and kept for $24 \mathrm{~h}$ before allowed to cool to room temperature. The reaction mixture was then filtered and washed by diethyl ethers through a silica pad to eliminate solid products. Organic layers were collected and evaporated to get the crude product which was then purified by silica gel column chromatography using petroleum ethers: ethyl acetate $(4: 1 \mathrm{v} / \mathrm{v})$ as eluent to obtain a yellow solid (150 mg, 51\% yield). ${ }^{1} \mathbf{H}$ NMR $(250 \mathrm{MHz}, \mathrm{DMSO}) \delta 8.63(\mathrm{~d}, J=2.3 \mathrm{~Hz}, 2 \mathrm{H}), 8.27$ (dd, $J=8.1,4.7 \mathrm{~Hz}, 6 \mathrm{H}), 8.15(\mathrm{dd}, J=8.7,2.4 \mathrm{~Hz}, 2 \mathrm{H}), 7.53-7.42(\mathrm{~m}, 8 \mathrm{H}), 7.37-7.28(\mathrm{~m}, 4 \mathrm{H})$. ${ }^{13} \mathrm{C}$ NMR $\left(62.5 \mathrm{MHz}, \mathrm{DMSO}-\mathrm{d}_{6}\right) \delta 178.4,140.3,136.2,135.8,132.0,129.8,126.9,126.6$, 123.4, 121.1, 109.9. HRMS (ESI+): calculated for $\mathrm{C}_{37} \mathrm{H}_{22} \mathrm{~N}_{2} \mathrm{OS}[\mathrm{M}+\mathrm{H}]^{+}$: 543.1453/found: 543.1525

\subsection{UV-visible absorption spectroscopy and colorimetric analysis}

UV-visible absorption spectra were acquired in DCM in a quartz cell at room temperature using a Jasco V-750 spectrophotometer. The molar extinction coefficients were determined using the Beer-Lambert law with experimental data obtained on solutions of known concentrations.

UV-visible transmission spectra were acquired from 200 to $800 \mathrm{~nm}$ on $1.4 \mathrm{~mm}$ thick polymer sample at room temperature using a Jasco V-750 spectrophotometer. Colorimetric analysis was given in the CIE $\mathrm{L}^{*} \mathrm{a}^{*} \mathrm{~b}^{*}$ colour system with the following parameters: a standard light D551, a 10 degrees standard observer, a $5 \mathrm{~nm}$ data interval and a colour matching function JIS Z8701:1999 with JIS Z8701:1999 and ASTM E308: 2008 as standard.

\subsection{Fluorescence experiment 2.4.1. Steady state fluorescence}

Fluorescence spectra were acquired in a quartz cell at room temperature using a JASCO ${ }^{\circledR} \mathrm{FP}-$ 750 spectrofluorometer. Excitation and emission spectra were recorded in DCM in a quartz cell.

\subsubsection{Time correlated single photon counting (TCSPC)}

The fluorescence excited state lifetimes were determined using a time-correlated single-photon counting system, a HORIBA ${ }^{\circledR}$ DeltaFlex with a HORIBA ${ }^{\circledR}$ PPD-850 as detector. The excitation source is a HORIBA ${ }^{\circledR}$ nanoLED-370 with an excitation wavelength of $367 \mathrm{~nm}$ and a pulse duration inferior to $1.4 \mathrm{~ns}$. The fluorescence intensity decay profiles were recorded in DCM in a quartz cell. A silica colloidal solution LUDOX $^{\circledR}$ was used to evaluate the impulse response function (IRF) of the apparatus.

\subsection{Laser flash photolysis (LFP)}

Nanosecond laser flash photolysis (LFP) experiments were performed using a Q-switched nanosecond Nd/YAG laser from Minilite Continuum ( $\lambda_{\mathrm{ex}}=355 \mathrm{~nm}, 9 \mathrm{~ns}$ pulses; energy reduced 
down to $10 \mathrm{~mJ}$ ) and an analysing system consisting of a pulsed xenon lamp, a monochromator, a fast photomultiplier, and a transient digitizer ${ }^{27}$. The decay traces were recorded in DCM in a quartz cell after a deoxygenation of the solution by $\mathrm{N}_{2}$ bubbling.

\subsection{Photopolymerisation kinetics (RT-FTIR)}

The experimental conditions for each photosensitive formulation are given in the captions of the figures. The weight percent of the photoinitiating system is calculated from the monomer content. The photoinitiator concentrations in each photosensitive formulation were chosen to ensure the same light absorption at $405 \mathrm{~nm}$.

All the polymerisations were performed at ambient temperature $\left(21-25^{\circ} \mathrm{C}\right)$ and irradiation was started at $\mathrm{t}=10 \mathrm{~s}$. A LED@405 nm (M405L3 - Thorlabs) having an intensity around 50 $\mathrm{mW} \cdot \mathrm{cm}^{-2}$ at the sample position was used for the photopolymerisation experiments. The emission spectrum is already available in the literature ${ }^{28}$.

A Jasco 4100 real-time Fourier transform infrared spectrometer (RT-FTIR) was used to follow the conversion of the acrylate functions of the TMPTA and of the epoxide group of EPOX. For the thin samples ( $\sim 25 \mu \mathrm{m}$ of thickness), the photosensitive formulations were deposited on polypropylene films under air for the cationic polymerisations of EPOX while the free radical polymerisations of TMPTA were performed in laminate (the formulation is sandwiched between two polypropylene films to reduce the $\mathrm{O}_{2}$ inhibition). The decrease of the $\mathrm{C}=\mathrm{C}$ double bond band or the epoxide group band was continuously monitored from 1581 to $1662 \mathrm{~cm}^{-1}$ or from 768 to $825 \mathrm{~cm}^{-1}$ respectively. For the thicker samples $(\sim 1.4 \mathrm{~mm}$ of thickness), the photocurable formulations were deposited on a polypropylene film inside a $1.4 \mathrm{~mm}$ thick mould under air. The evolutions of the $\mathrm{C}=\mathrm{C}$ double bond band and the epoxide group band were continuously followed from 6117 to $6221 \mathrm{~cm}^{-1}$ and from 3710 to $3799 \mathrm{~cm}^{-1}$ respectively.

\subsection{Computational Procedure}

Molecular orbital calculations were carried out using the Gaussian 03 package. The electronic absorption spectrum of TX-2CBZ, TX-2DPA, TX-2PTZ and ITX were calculated from the time-dependent density functional theory at the RTD-mPW1PW91-FC/6-31G* level on the relaxed geometries calculated at the $\mathrm{uB} 3 \mathrm{LYP} / 6-31 \mathrm{G}^{*}$ level. The geometries were frequencychecked $^{29}$.

\subsubsection{D laser writing experiments}

The photosensitive resin was deposited onto a microscope slide ( $1 \mathrm{~mm}$ thick sample). A computer-controlled laser diode at $405 \mathrm{~nm}$ (size of the irradiation spot around $50 \mu \mathrm{m}$ ) was used for the spatially controlled irradiation. The generated pattern was analyzed by a numerical optical microscope (DSX-HRSU from OLYMPUS Corp)

\section{Results and discussion 3.1. Photoinitiator synthesis}

The synthetic routes toward the different thioxanthone derivatives are outlined in Scheme 1. 


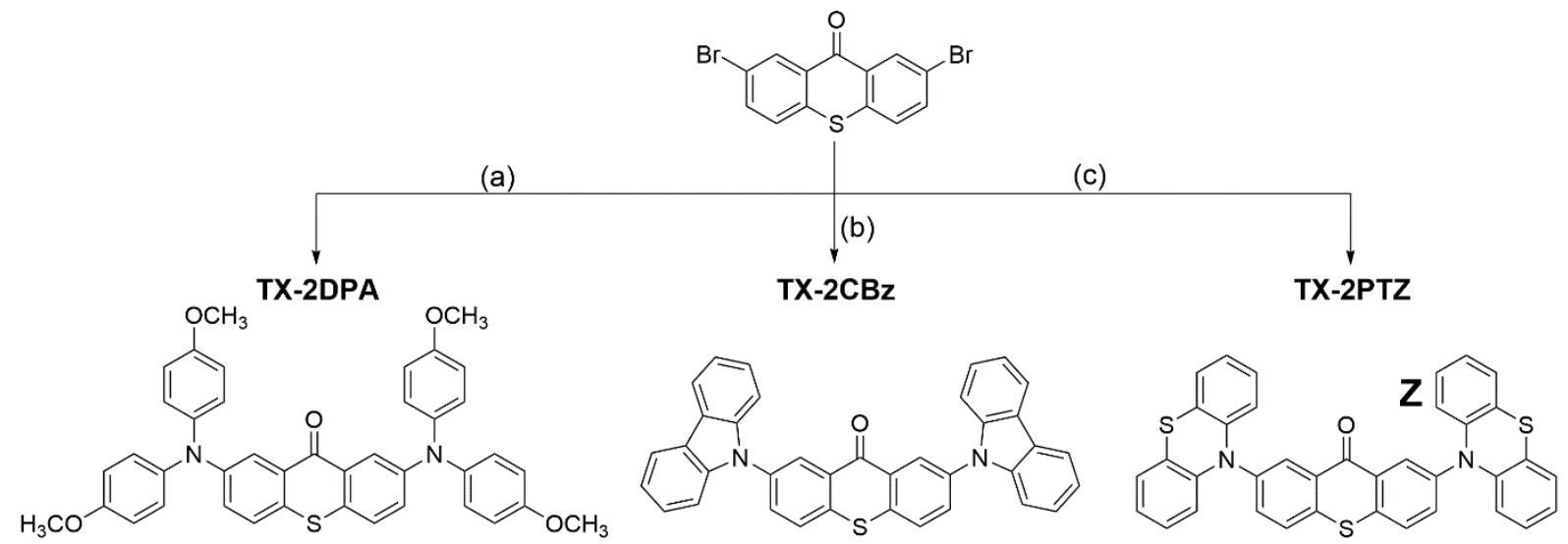

Scheme 1: Synthesis of thioxanthone-based photoinitiators. Reagents and conditions (a) di(4methoxyphenyl)amine, $\mathrm{NaO} t \mathrm{Bu}, \mathrm{PdCl}_{2}\left(\mathrm{PPh}_{3}\right)_{4}$, Toluene, $110^{\circ} \mathrm{C}$, 24h. (b) Carbazole, $\mathrm{Na}_{2} \mathrm{CO}_{3}$, CuI, 18-crown-6, Nitrobenzene, $160^{\circ} \mathrm{C}$, 24h. (c) phenothiazine, $\mathrm{NaO} t \mathrm{Bu}, \mathrm{PdCl}_{2}\left(\mathrm{PPh}_{3}\right)_{4}$, Toluene, $110^{\circ} \mathrm{C}, 24 \mathrm{~h}$.

Briefly, all compounds were synthetized in one step from 2,7-dibromo-9H-thioxanthen-9-one ${ }^{26}$ and the corresponding diarylamine using twofold $\mathrm{C}-\mathrm{N}$ coupling reactions. While TX-2DPA and TX-2PTZ were obtained by using Pd-catalyzed Buchwald-Hartwig amination, the coppercatalyzed Ullmann amination was used to afford TX-2CBZ.

\subsection{Photochemical properties}

\subsubsection{Ground state: $U V$-visible absorption and molecular modelling}

Absorption spectra of the selected thioxanthone derivatives (Figure 1) and of ITX in dichloromethane are depicted in Figure 3. TX-2CBZ, TX-2DPA, TX-2PTZ and ITX show mainly an ultraviolet absorption. However, their lowest energy transition corresponds to a near UV-visible absorption. The longest absorption wavelength and corresponding molar extinction coefficient for the four thioxanthone derivatives are gathered in Table 1. Interestingly for the three investigated thioxanthones TX-2CBZ, TX-2DPA and TX-2PTZ, the lowest energy transition is shifted toward longer wavelengths depending on the electron donating substituent. With the dimethoxydiphenylamine (DPA) substituents, the absorption peak is more shifted than with carbazole (CBZ) or phenothiazine (PTZ) substituents. The emission spectrum of the LED at $405 \mathrm{~nm}$ shows a fine overlap with the light absorption of the thioxanthone derivatives (Figure 3 inset). Indeed, TX-2CBZ, TX-2DPA, TX-2PTZ and ITX possess molar extinction coefficients at $405 \mathrm{~nm}$ around 5900, 2000, 2600 and $1000 \mathrm{~L} \cdot \mathrm{mol}^{-1} \cdot \mathrm{cm}^{-1}$ respectively. Thus, the three investigated thioxanthone derivatives present a bathochromic shift compared to ITX with higher extinction coefficients. For a better understanding of these light absorption properties, molecular orbital calculations were carried out. The wavelengths determined for the lowest energy transition by theoretical calculations (Table 1) are rather coherent with the experimental ones. 


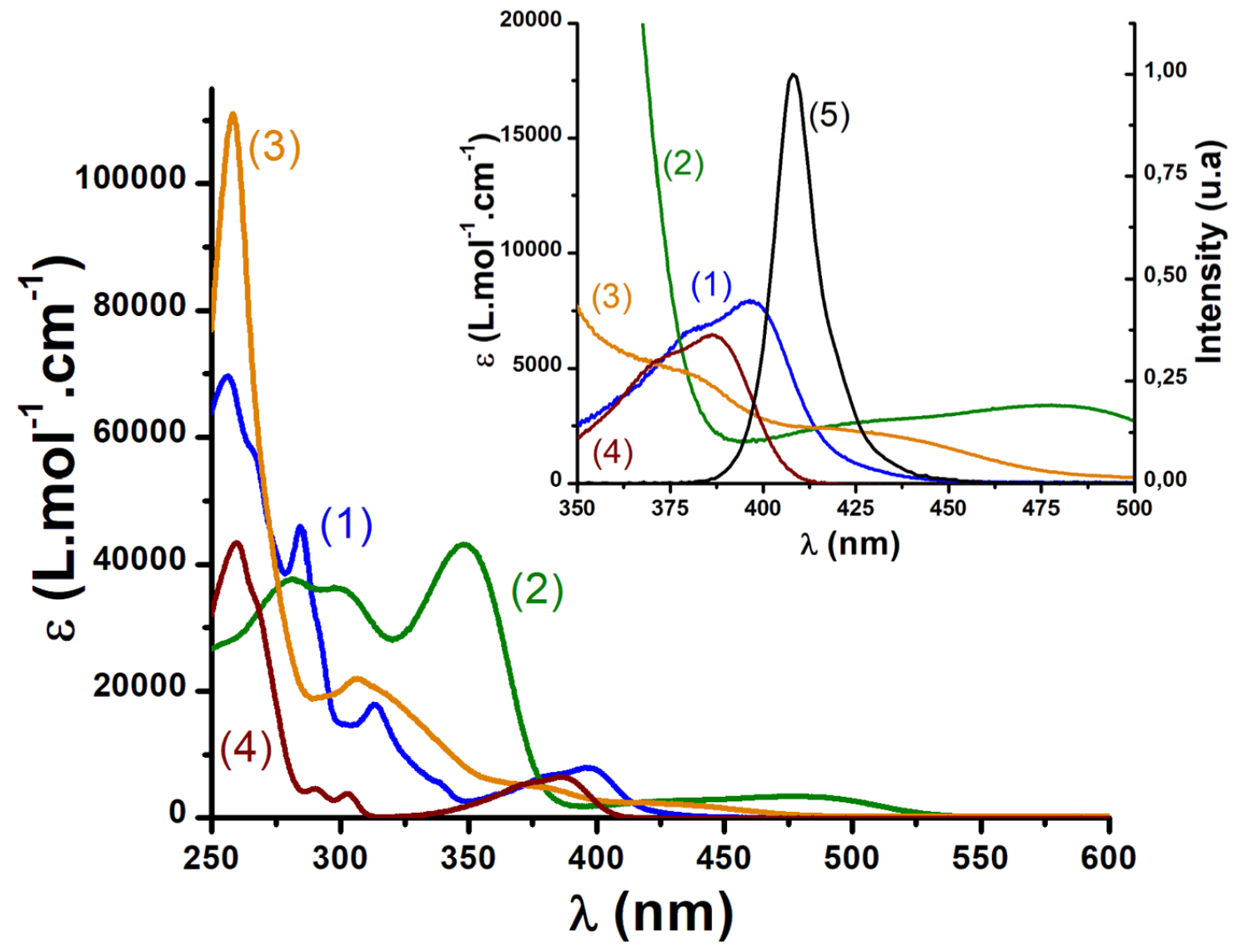

Figure 3: Absorption spectra of (1) TX-2CBZ, (2) TX-2DPA, (3) TX-2PTZ and (4) ITX in dichloromethane. The inset is a zoom of the absorption spectra overlaid with (5) the normalized emission spectra of the used LED at $405 \mathrm{~nm}$.

Table 1: Absorption properties of the different thioxanthone derivatives.

\begin{tabular}{lcccc}
\hline & $\lambda_{\max }(\mathrm{nm})$ & $\varepsilon \lambda_{\max }\left({\left.\mathrm{L} . \mathrm{mol}^{-1} . \mathrm{cm}^{-1}\right)}\right.$ & $\varepsilon_{405 \mathrm{~nm}}\left(\mathrm{~L} \cdot \mathrm{mol}^{-1} \cdot \mathrm{cm}^{-1}\right)$ & $\lambda_{\text {max calculated }}(\mathrm{nm})$ \\
\hline ITX & 386 & $6.5 \times 10^{3}$ & $1.0 \times 10^{3}$ & 340 \\
TX-2CBZ & 396 & $7.9 \times 10^{3}$ & $5.9 \times 10^{3}$ & 428 \\
TX-2DPA & 478 & $3.4 \times 10^{3}$ & $2.0 \times 10^{3}$ & 470 \\
TX-2PTZ & $305 ; 415^{\mathrm{a}}$ & $2.4 \times 10^{3}$ & $2.6 \times 10^{3}$ & 335
\end{tabular}

${ }^{a}$ shouldered band

The optimised geometries and frontier orbitals, energies of the highest occupied molecular orbital (HOMO) and lowest unoccupied molecular orbital (LUMO) were determined by theoretical calculations, using density functional theory at uB3LYP/6-31G* level of the theory. The contour plots of the optimised geometries frontier orbitals are depicted in Figure 4. Interestingly, for TX-2CBZ, TX-2DPA, TX-2PTZ and ITX, the electronic density of the LUMO is almost entirely located on the thioxanthone moiety while the substituents are participating in the HOMO. This complete separation between the frontier orbitals suggests a 
charge transfer behaviour confirming the D-A-D structure. This charge transfer behaviour is coherent with the shift of the absorption spectra observed upon modification of the electron donating groups. Another important parameter for the photochemical reactivity of thioxanthone is usually the triplet energy level. The computed thioxanthone derivatives triplet state $T_{1}$ energies are gathered in Figure 4. The energy of the triplet state decreases in the series ITX > $\mathrm{TX}-2 \mathrm{CBZ} \approx \mathrm{TX}-2 \mathrm{PTZ}>\mathrm{TX}-2 \mathrm{DPA}$.

\section{\begin{tabular}{lll}
\hline HOMO & LUMO & $\mathrm{E}_{\mathrm{T}_{1}}\left(\text { kcal.mol }^{-1}\right)^{\mathrm{a}}$ \\
\hline
\end{tabular} \\ TX-2CBZ
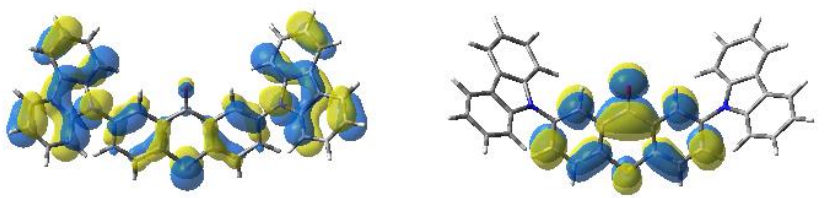 \\ 56.38 \\ TX-2DPA
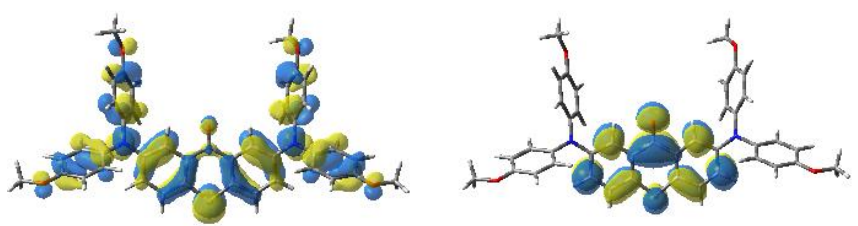 \\ TX-2PTZ
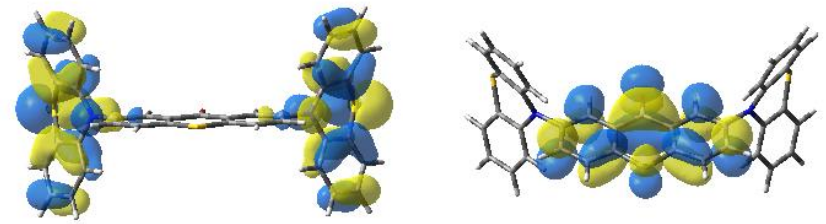 \\ ITX
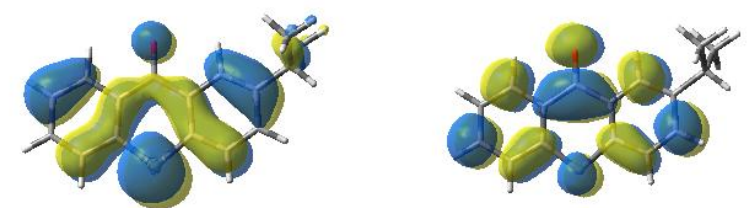

Figure 4: Contour plots of the frontier orbitals determined for the different thioxanthone derivatives examined in this work and optimised at the B3LYP/6-31G* level of theory and their calculated $\mathrm{T}_{1}$ triplet state energy, ${ }^{\mathrm{a}}$ level $\pm 3 \mathrm{kcal}^{\mathrm{mol}}{ }^{-1}$

\subsubsection{Singlet excited state: Steady state and time resolved fluorescence}

To assess the properties related to the $\mathrm{S}_{1}$ singlet excited states of the TX-2CBZ, TX-2DPA, TX2PTZ and ITX, a steady state fluorescence analysis in dichloromethane was performed. Unfortunately, no fluorescence properties are observed for TX-2DPA and TX-2PTZ. This absence of fluorescence may be associated to a deactivation of the lowest singlet state by a nonradiative process such as an internal conversion or an intersystem crossing. The excitation and emission spectra of TX-2CBZ are presented in Figure 5. The fluorescence emission of this thioxanthone derivative is centred at $502 \mathrm{~nm}$ which is consistent with previous results ${ }^{25}$. The formation of a fluorescent photoproduct is also observed in Figure 5 with an emission centred at $420 \mathrm{~nm}$ and partially overlapping with the thioxanthone derivative emission peak. This photoproduct is associated with an impurity since the excitation spectrum at $420 \mathrm{~nm}$ does not fit with the absorption spectrum of TX-2CBZ. In order to evaluate the energy of the first singlet excited state $S_{1}$, an extrapolation of the shape of the emission peak as a gaussian function (dash 
line curve) was required. From the crossing point of the absorption and emission spectra, the energy of the first singlet excited state $S_{1}$ is estimated to be around $66.9 \mathrm{kcal}^{-\mathrm{mol}^{-1}}$ which is coherent with the $66.6 \mathrm{kcal}^{\mathrm{mol}}{ }^{-1}$ calculated with molecular modelling of the absorption spectrum. The energetic gap between the triplet state $\mathrm{T}_{1}$ and the singlet state $\mathrm{S}_{1}$ of TX-2CBZ is between 5 to $15 \mathrm{kcal}^{\mathrm{mol}}{ }^{-1}$ which is higher than the energy brought by the medium due to thermal agitation ${ }^{30}$. This last result which come from computational calculation suggests that TX-2CBZ does not present a thermally activated delayed fluorescence (TADF) behaviour but only an extended singlet state lifetime which is in accordance to literature ${ }^{25}$. However, influence of the solvent which could induce the stabilisation or the destabilisation of TX-2CBZ fundamental and excited states, was not considered here. Thus, the energetic gap between the triplet state $T_{1}$ and the singlet state $S_{1}$ could be smaller and TX-2CBZ could present a thermally activated delayed fluorescence (TADF) behaviour in other media.

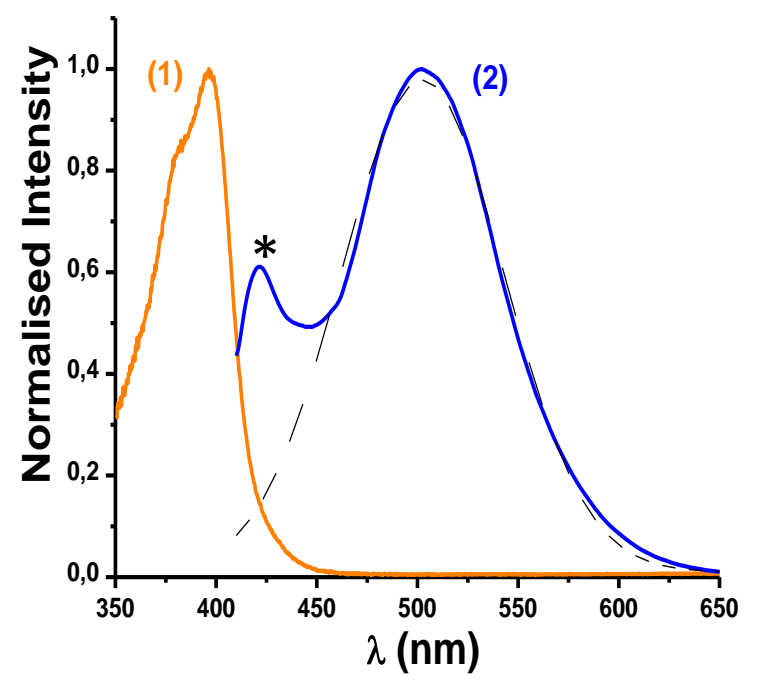

Figure 5: Fluorescence spectra of TX-2CBZ in dichloromethane under air [TX-2CBZ] $=1.4 \times$ $10^{-5}$ mol. $\mathrm{L}^{-1}$ (1) excitation spectrum $\lambda_{\mathrm{em}}=502 \mathrm{~nm}$, (2) emission spectrum $\lambda_{\mathrm{ex}}=400 \mathrm{~nm}$, * peak associated with an impurity.

Analysis by time-correlated single-photon counting (TCSPC) of the $\mathrm{S}_{1}$ lifetimes shows in Figure 6 the decay profile of the TX-2CBZ fluorescence. From mono-exponential curve fitting of the decay profile, the first singlet state lifetime is assessed at $7 \mathrm{~ns} \pm 1 \mathrm{~ns}$. In comparison, the first singlet state lifetime of ITX is much shorter, inferior to $1.4 \mathrm{~ns}$ which is the minimal resolution of the experimental setup; this is in agreement with the singlet state lifetime of 200 ps evaluated in the literature in acetonitrile ${ }^{31}$. Fluorescence decay profile of ITX obtained by time-correlated single-photon counting (TCSPC) is presented in Supporting Information Figure $\mathrm{S} 1$. The strong elongation of the first singlet state lifetime in TX-2CBZ vs. ITX could be a consequence of the rigidification of the molecule structure by the carbazole substituents. 


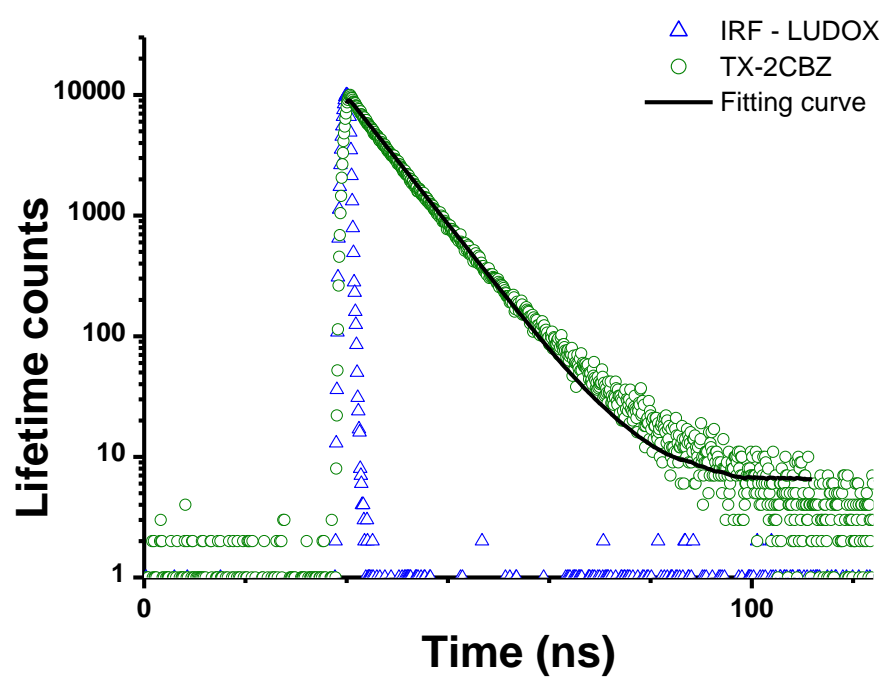

Figure 6: Time-correlated single-photon counting of TX-2CBZ in dichloromethane, $\lambda_{\mathrm{ex}}=367$ $\mathrm{nm}, \lambda_{\mathrm{em}}=502 \mathrm{~nm}$ and $[\mathrm{TX}-2 \mathrm{CBZ}]=1.4 \times 10^{-5} \mathrm{~mol} \cdot \mathrm{L}^{-1}$.

In order to fully characterise the reactivity of the singlet state of TX-2CBZ toward amine (EDB) or iodonium salt (Iod), steady state fluorescence quenching was performed in dichloromethane by gradually adding EDB or Iod in a TX-2CBZ solution. The Stern-Volmer quenching plots are presented in Figure S2 in SI. What is significant about these results is the slope of the linear regression of the Stern-Volmer plot which corresponds to the product of the observed rate constant of quenching $\mathrm{k}_{\mathrm{q}}$ and the singlet lifetime $\tau_{0}$. The observed rate constants of quenching $\mathrm{k}_{\mathrm{q}}$ are assessed at $6.5 \times 10^{9}$ and $4.7 \times 10^{9} \mathrm{~L}^{\mathrm{mol}}{ }^{-1} . \mathrm{s}^{-1}$ for TX-2CBZ/Iod and TX-2CBZ/EDB respectively. These constants are very high albeit slightly smaller than the diffusional rate constant, $\mathrm{k}_{\mathrm{d}}=1.5 \times 10^{10} \mathrm{~L} \cdot \mathrm{mol}^{-1} \cdot \mathrm{s}^{-1} 32$. Thus, these reactions are really efficient and almost diffusion-controlled.

\subsubsection{Triplet excited state: Laser flash photolysis}

Laser flash photolysis experiments were also carried out on the different thioxanthone derivatives in order to characterise their triplet state $T_{1}$. Analogously to the fluorescence analysis, the triplet state of TX-2DPA and TX-2PTZ were not detected in laser flash photolysis experiments. This result may be explained by either an absence of intersystem crossing or a triplet excited state lifetime below the resolution of our LFP spectrometer (<10ns) or a nonradiative deexcitation of the triplet state. The decay traces of the $\mathrm{T}_{1}$ for TX-2CBZ and ITX are presented along with their transient absorption spectra recorded $5.12 \mu \mathrm{s}$ and $10.24 \mu$ s after the acquisition start (laser pulse for $\mathrm{t}=5 \mu \mathrm{s}$ and $\mathrm{t}=10 \mu \mathrm{s}$ respectively) in Figure 7 and Figure 8 respectively. The transient spectra of TX-2CBZ and ITX present an absorption from $570 \mathrm{~nm}$ to $670 \mathrm{~nm}$ which is typically assigned to thioxanthone triplet state ${ }^{33}{ }^{34}$. The triplet state lifetime of TX-2CBZ and ITX in deoxygenated dichloromethane are about $1.0 \mu \mathrm{s}$ and $5.8 \mu \mathrm{s}$. Thus, the triplet excited state lifetimes of both TX-2CBZ and ITX are of the same order of magnitude. 

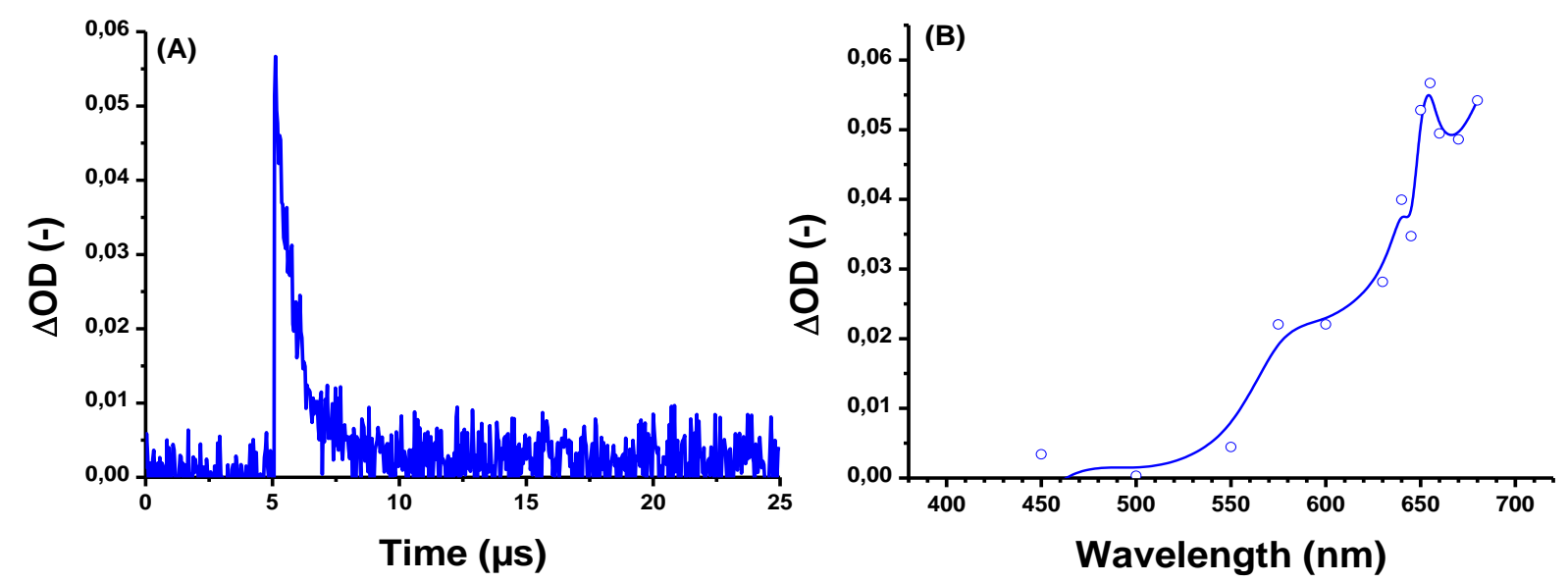

301

Figure 7: (A) Decay trace of TX-2CBZ recorded at $655 \mathrm{~nm}$ under nitrogen in dichloromethane after laser excitation at $355 \mathrm{~nm}$ laser pulse for $\mathrm{t}=5 \mu \mathrm{s}(\mathrm{B})$ Transient absorption spectrum of TX$2 \mathrm{CBZ}$ recorded after the laser pulse for $\mathrm{t}=5.12 \mu \mathrm{s}$
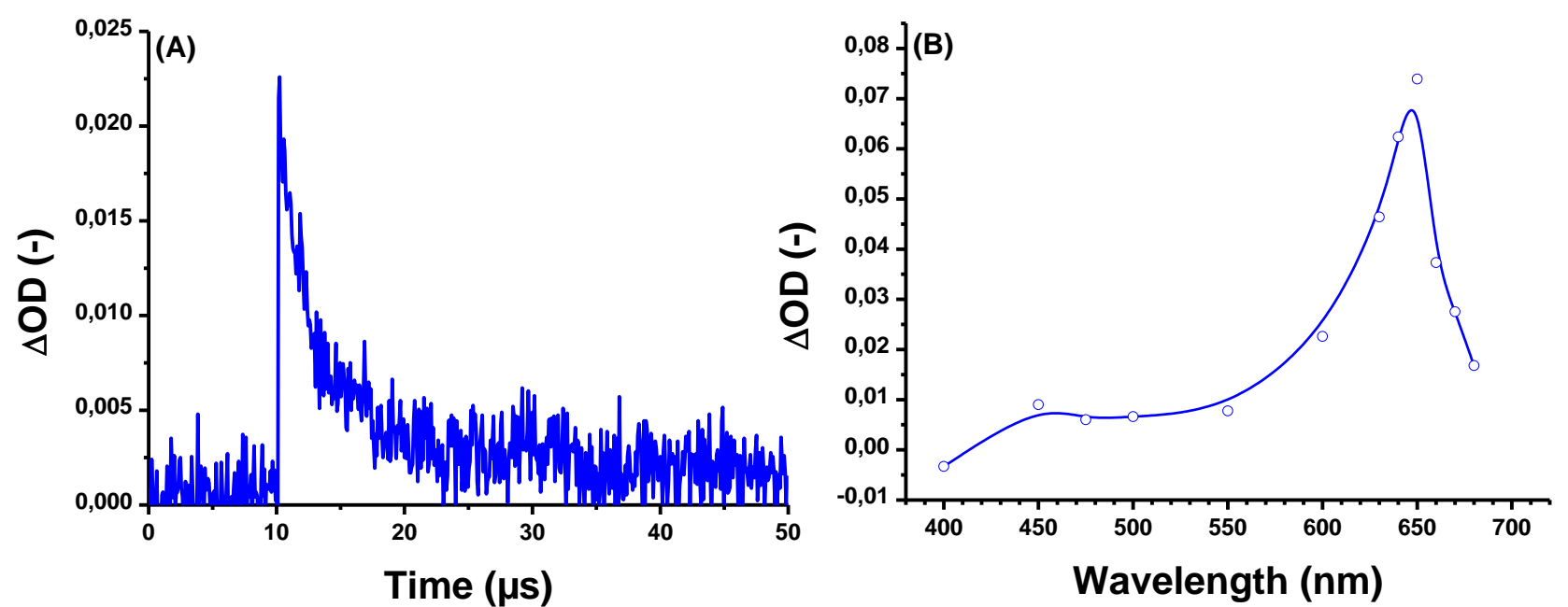

Figure 8: (A) Decay trace of ITX recorded at $650 \mathrm{~nm}$ under nitrogen in dichloromethane after laser excitation at $355 \mathrm{~nm}$ laser pulse for $\mathrm{t}=10 \mu \mathrm{s}$ (B) Transient absorption spectrum of ITX recorded after the laser pulse for $\mathrm{t}=10.24 \mu \mathrm{s}$

In order to fully characterise the reactivity of the triplet state of TX-2CBZ toward amine (EDB) or iodonium salt (Iod), triplet quenching was performed in dichloromethane by gradually adding EDB or Iod in a TX-2CBZ solution monitoring the change of triplet state decays. The Stern-Volmer quenching plots are presented in Figure S3 in SI. The observed rate constant of quenching $\mathrm{k}_{\mathrm{q}}$ are assessed at $7.79 \times 10^{8}$ and $5.44 \times 10^{9} \mathrm{~L} . \mathrm{mol}^{-1} . \mathrm{s}^{-1}$ for TX-2CBZ/Iod and TX2CBZ/EDB respectively. Thus, the reactions from TX-2CBZ triplet state are really efficient and close to the diffusion limit.

Since both $S_{1}$ and $T_{1}$ have a similar reactivity toward the additives (EDB or Iod), we focus on the different modes of deactivation of the excited state $S_{1}$ in order to determine which excited state is involved in the polymerisation initiating step. 
The different deactivation processes of the first singlet state were characterised through their specific quantum yields (Scheme 2).

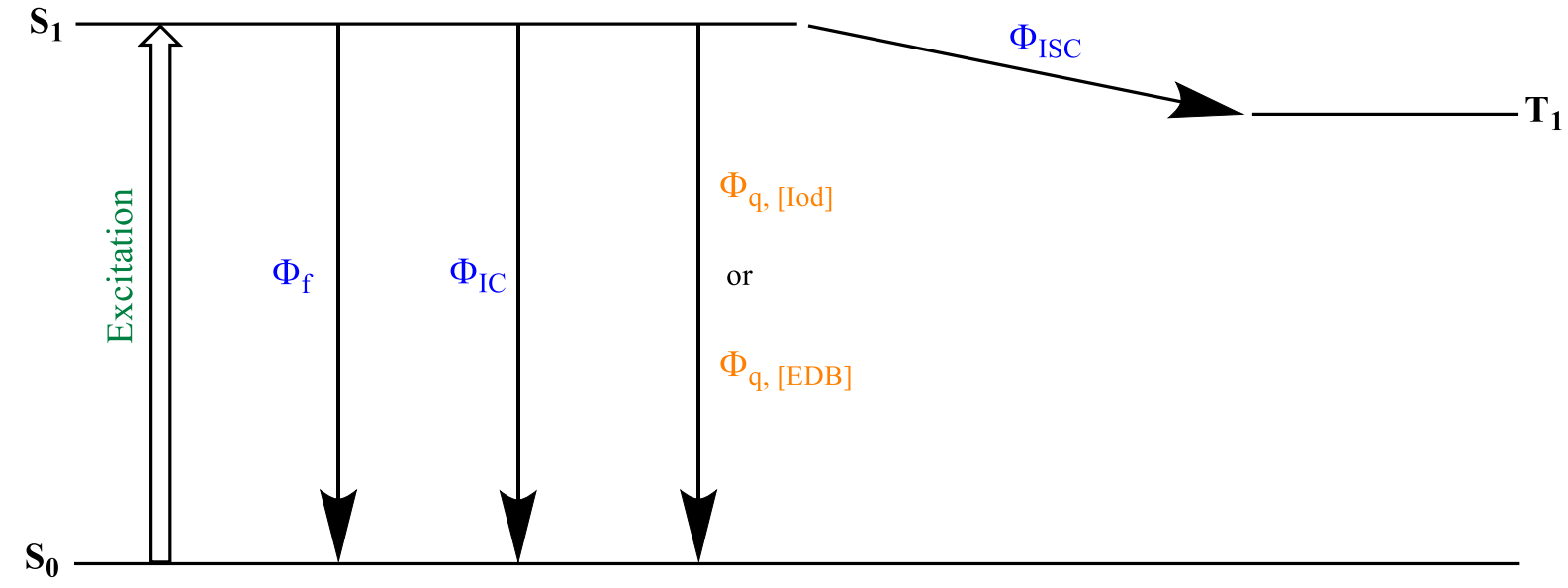

Scheme 2: Processes from the $S_{1}$ excited state: $\Phi_{\mathrm{f}}, \Phi_{\mathrm{IC}}, \Phi_{\mathrm{q},[\mathrm{Iod}]}, \Phi_{\mathrm{q},[\mathrm{EDB}]}$ and $\Phi_{\mathrm{ISC}}$ represent the fluorescence quantum yield, the internal conversion quantum yield, the quantum yield of quenching by Iod, the quantum yield of quenching by EDB and the intersystem crossing quantum yield.

As shown in Scheme 2, the deactivation of the singlet state occurs through the competition between up to four processes: internal conversion (IC), fluorescence (f), intersystem crossing (ISC) and quenching (Q). To simplify the study, TX-2CBZ was first considered in dichloromethane without any quencher. In this case, the quantum yield of the intersystem crossing, fluorescence and internal conversion could be defined by the formulas presented in Equations 1-4 ${ }^{35}$.

$$
\begin{aligned}
& \Phi_{I S C}=\frac{k_{I S C}}{k_{I S C}+k_{f}+k_{I C}}=k_{I S C} \tau_{0} \\
& \Phi_{f}=\frac{k_{f}}{k_{I S C}+k_{f}+k_{I C}}=k_{f} \tau_{0} \\
& \Phi_{I C}=\frac{k_{I C}}{k_{I S C}+k_{f}+k_{I C}}=k_{I C} \tau_{0} \\
& \Phi_{I S C}+\Phi_{f}+\Phi_{I C}=1
\end{aligned}
$$

In these equations, $\Phi_{\mathrm{ISC}}, \Phi_{\mathrm{f}}, \Phi_{\mathrm{IC}}, \mathrm{k}_{\mathrm{ISC}}, \mathrm{k}_{\mathrm{f}}, \mathrm{k}_{\mathrm{IC}}$ and $\tau_{0}$ represent the quantum yield of intersystem crossing, the quantum yield of fluorescence, the quantum yield of internal conversion, the rate constant for intersystem crossing, the rate constant of fluorescence, the rate constant for internal conversion $\mathrm{S}_{1} \rightarrow \mathrm{S}_{0}$ and the singlet lifetime in the absence of any quencher, respectively.

The quantum yield of the intersystem crossing was evaluated through laser flash photolysis experiments. The quantum yield of intersystem crossing was determined from the transient 
yields of triplet 1-methylnaphthlene produced when it was added as a quencher. Benzophenone was used as standard due to his well-known intersystem crossing quantum yield $\Phi_{\text {ISC }}=1^{32}$. The decay trace of TX-2CBZ and benzophenone in presence of 1-methylnaphthalene are presented in Figure S4 in SI. The intersystem crossing quantum yield of TX-2CBZ is about $0.60 \pm 0.03$.

To complete this assessment, the quantum yield of fluorescence was determined by comparing the integrated areas beneath the fluorescence emission spectra of TX-2CBZ with that of a standard. The emission spectra were recorded with the same acquisition condition. Quinine sulphate in $\mathrm{H}_{2} \mathrm{SO}_{4} 0.1 \mathrm{M}$ was used as a standard with a fluorescence quantum yield $\Phi_{\mathrm{f}}=0.54{ }^{36}$. Variation of the integrated fluorescence intensity vs absorbance at the excitation wavelength is presented in Supporting Information Figure S5. The fluorescence quantum yield of TX-2CBZ is assessed at 0.03. Since the intersystem crossing quantum yield of TX-2CBZ is about 0.60 , in absence of quenching reaction, the internal conversion quantum yield could be inferred from the intersystem crossing quantum yield and the fluorescence quantum yield of TX-2CBZ and is assessed at 0.37 .

Thus, the deactivation of the singlet excited state while omitting the quenching reactions seems to be a competition between internal conversion and intersystem crossing. However, in presence of the additives of the two-component initiating system, Iod or EDB, the quenching reaction could not be neglected. The singlet quenching quantum yields were derived from the observed rate constant of quenching $\mathrm{k}_{\mathrm{q}}$ using Equation 5. The quenching quantum yield is about 0.65 and 0.77 for system TX-2CBZ/Iod and TX-2CBZ/EDB respectively. Using the Equation 6 with a quencher concentration in accordance with the conditions of the polymerisation experiments, the influence of the quenching on the quantum yield $\Phi_{\mathrm{f}}$, $\Phi_{\mathrm{IC}}$ and $\Phi_{\mathrm{ISC}}$ was determined. These recalculated quantum yields are $\Phi_{q,[\text { Iod }]}=0.65, \Phi_{f,[\text { Iod }]}=0.01$, $\Phi_{I C,[I o d]}=0.13$ and $\Phi_{I S C,[I o d]}=0.21$ in presence of Iod (Figure S7 in SI), and in presence of $\mathrm{EDB}, \Phi_{q,[E D B]}=0.77, \Phi_{f,[E D B]}<0.01, \Phi_{I C,[E D B]}=0.09$ and $\Phi_{I S C,[E D B]}=0.14$. In presence of either additive the quenching of TX-2CBZ became the main deactivation mode of the first singlet excited state.

$$
\Phi_{q,[Q]}=\frac{k_{q} \tau_{0}[Q]}{1+k_{q} \tau_{0}[Q]}
$$

In this equation, $\Phi_{q,[Q]}, k_{q},[Q]$ and $\tau_{0}$ represent the quenching quantum yield, the observed rate constant of quenching, the quencher concentration and the singlet lifetime, respectively.

$$
\frac{\Phi_{X}}{\Phi_{X,[Q]}}=1+k_{q} \tau_{0}[Q]
$$

In this equation, $\Phi_{X,[Q]}, \Phi_{X}, k_{q},[Q]$ and $\tau_{0}$ represent the quantum yield in presence or absence of a quencher Q (where X stands for either ISC, f or IC), the observed rate constant of quenching, the quencher concentration and the singlet lifetime, respectively.

All the thioxanthone derivatives possess absorption properties making them interesting candidates for the photoinitiation under visible light. The reactivity of TX-2CBZ with Iod or EDB occurs through the first singlet excited state while for isopropylthioxanthone this reactivity usually occurs through the first triplet excited state ${ }^{37}$. Since the properties of the 
thioxanthone derivatives have been assessed, we focus on their use as photoinitiating systems for the polymerisation of various monomers.

\subsection{Polymerisation}

\subsubsection{Free radical polymerisation in TMPTA}

Thioxanthone derivatives were investigated as photoinitiators for the free radical polymerisation of TMPTA upon LED irradiation at $405 \mathrm{~nm}$ to produce either $25 \mu \mathrm{m}$ thick samples in laminate or $1.4 \mathrm{~mm}$ thick samples under air.

TX-2CBZ, TX-2DPA and TX-2PTZ were tested as type II photoinitiators with an amine (EDB) and comparisons were established with a benchmark commercial initiator, 2isopropylthioxanthone (ITX). After the irradiation of the EDB/thioxanthone derivative system, a reaction took place between the excited state of the thioxanthone derivative and EDB. Provided that these derivatives react as unsubstituted thioxanthone (TX), an electron transfer from EDB ground state to thioxanthone excited state followed by a rapid proton transfer will occurred as stated in reaction $\mathrm{r} 1-\mathrm{r} 2{ }^{38}$. The polymerisation profiles of TMPTA in presence of a photoinitiator/EDB system are presented in Figure 9. Among the tested thioxanthone derivatives, only ITX and TX-2CBZ allow the formation of a polymer with a tack-free surface. For the polymerisation of $25 \mu \mathrm{m}$ thick samples (Figure 9A), only ITX and TX-2CBZ systems show a high efficiency and fast kinetics while TX-2DPA and TX-2PTZ systems demonstrate a low efficiency and slow kinetics. The final $\mathrm{C}=\mathrm{C}$ double bond conversions are 53\%, 36\%, $19 \%$ and 7\% for ITX, TX-2CBZ, TX-2DPA and TX-2PTZ respectively. The same trend in the polymerisation efficiency is observed for $1.4 \mathrm{~mm}$ thick samples (Figure 9B). However, a very high efficiency and fast kinetics were observed for ITX/EDB and TX-2CBZ/EDB (final conversion $=83 \%$ and $84 \%$ respectively). These results suggested that the thioxanthone derivatives react similarly to the reference ITX. Moreover, the poor efficiency of TX-2DPA and TX-2PTZ are in accordance with the non-measurable singlet/triplet state lifetime suggesting probably mainly internal conversion as a deactivation pathway of the S1 excited state with a slow reaction with Iod or EDB.

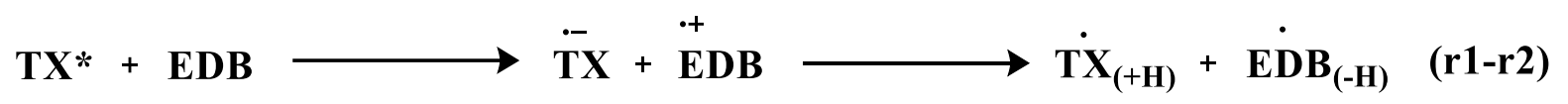

TX-2CBZ, TX-2DPA and TX-2PTZ were also tested as photoinitiator with an iodonium salt (Iod). For the sake of comparison, polymerisation was also performed with the standard system ITX/Iod. The thioxanthone derivative/Iod interaction corresponds to an electron transfer reaction which leads to an aryl radical Ar as presented in reactions $\mathrm{r} 3-\mathrm{r} 4{ }^{39}$. The polymerisation profiles of TMPTA in presence of a photoinitiator/Iod system are presented in Figure 10. For the polymerisation of $25 \mu \mathrm{m}$ thick sample (Figure 10A), the efficiency trend of thioxanthone derivative as photoinitiator respects the following order: ITX $>$ TX-2CBZ $>$ TX-2PTZ $>$ TX2DPA. The final $\mathrm{C}=\mathrm{C}$ double bond conversions are $63 \%, 46 \%, 38 \%$ and $23 \%$ respectively and a polymer with a tack-free surface is only obtained with ITX, TX-2CBZ and TX-2PTZ. Similar results in terms of the polymerisation efficiency are obtained for $1.4 \mathrm{~mm}$ thick samples (Figure 10B). Among the tested thioxanthone derivatives, only ITX allows to obtain a polymer with a tack-free surface while TX-2CBZ and TX-2PTZ allow to synthesize a polymer with a close to tack-free surface. The final $\mathrm{C}=\mathrm{C}$ double bond conversions are $74 \%, 78 \%, 51 \%$ and $25 \%$ with ITX/Iod, TX-2CBZ/Iod, TX-2PTZ/Iod and TX-2DPA/Iod respectively. Since the incertitude on the conversion was of $3 \%$ in these conditions, the efficiency trend of thioxanthone derivatives as photoinitiators respects the following order: TX-2CBZ $\approx$ ITX $>$ TX-2PTZ $>$ TX- 
2DPA. This last order of reactivity is in accordance with the one of the triplet state energy found by molecular modelling.

427

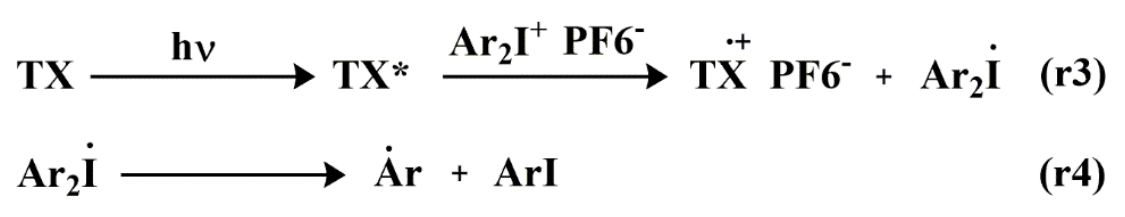

Among the investigated thioxanthone derivatives used for the free radical polymerisation of TMPTA, TX-2CBZ appears as an interesting photoinitiator. Indeed, in initiating system with either the amine EDB or the iodonium salt Iod, TX-2CBZ exhibits performances slightly lower or similar than the standard photoinitiator ITX but for a lower weight percentage in the formulation as the comparison was given for similar absorption properties at $405 \mathrm{~nm}$. When the same molar percentage is used in the formulation, the same performances are observed as showed in Figure S6 in SI.

(A)

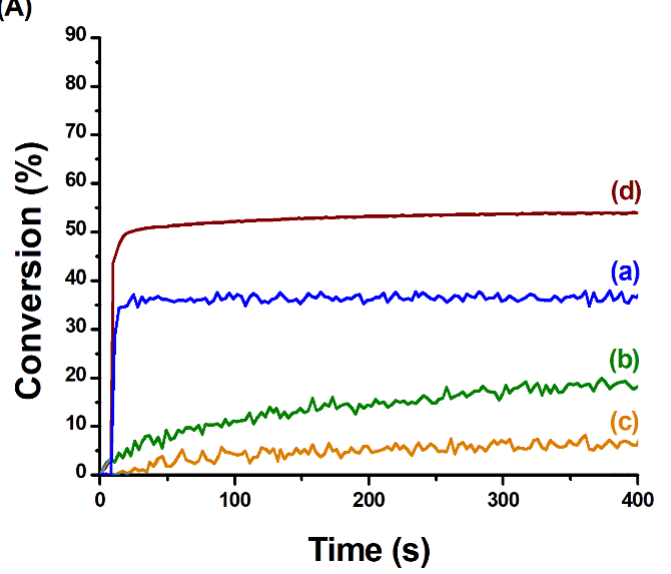

(B)

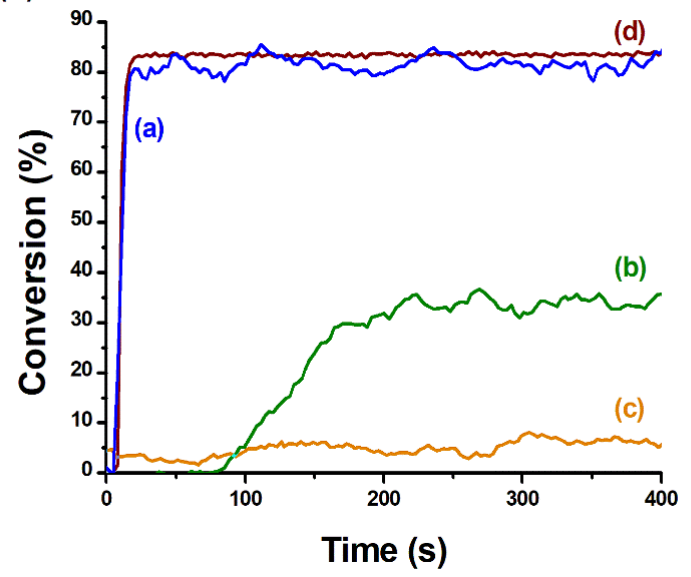

Figure 9: Polymerisation profiles (acrylate function conversion vs irradiation time) of TMPTA upon irradiation with a LED at $405 \mathrm{~nm}$, irradiation starts at $10 \mathrm{~s}, 50 \mathrm{~mW} . \mathrm{cm}^{-2}$; (A) sample thickness $=25 \mu \mathrm{m}$ and (B) sample thickness $=1.4 \mathrm{~mm}$. Photoinitiating systems: (curve a) TX2CBZ/EDB (0.07/1.9 w/wt\%), (curve b) TX-2DPA/EDB $(0.22 / 1.9 \mathrm{w} / \mathrm{wt} \%$ ), (curve c) TX2PTZ/EDB (0.17/2.0 w/wt $\%)$ and (curve d) ITX/EDB (0.19/2.0 w/wt $\%)$ 
(A)

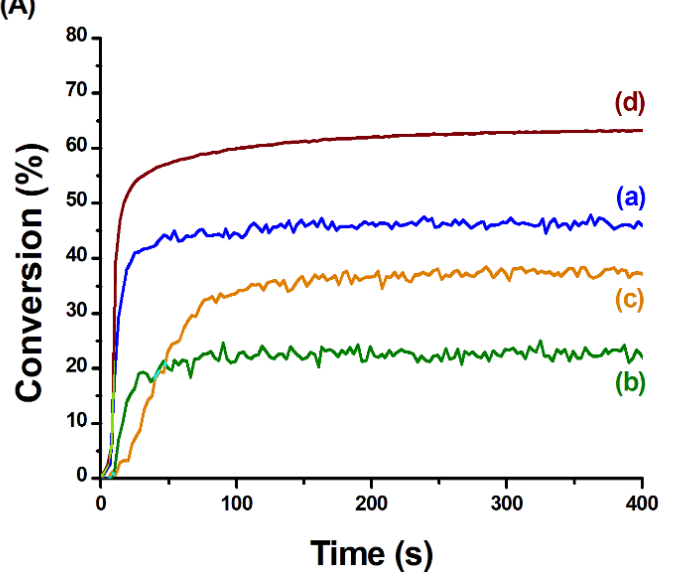

(B)

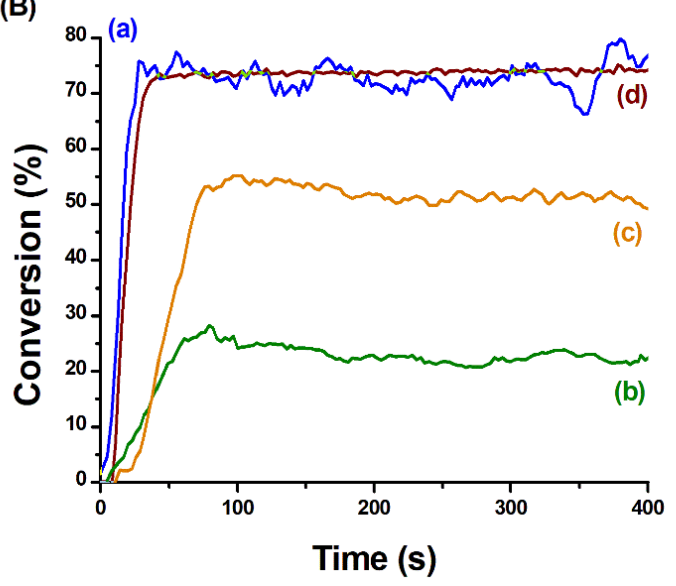

443

444

445

446

Figure 10: Polymerisation profiles (acrylate function conversion vs irradiation time) of TMPTA upon irradiation with a LED at $405 \mathrm{~nm}$, irradiation starts at $10 \mathrm{~s}, 50 \mathrm{~mW} . \mathrm{cm}^{-2}$; (A) sample thickness $=25 \mu \mathrm{m}$ and (B) sample thickness $=1.4 \mathrm{~mm}$. Photoinitiating systems: (curve a) TX2CBZ/Iod $(0.07 / 1.9 \mathrm{w} / \mathrm{wt} \%)$, (curve b) TX-2DPA/Iod $(0.22 / 2.0 \mathrm{w} / \mathrm{wt} \%$ ), (curve c) TX2PTZ/Iod $(0.18 / 2.0 \mathrm{w} / \mathrm{wt} \%)$ and (curve d) ITX/Iod $(0.19 / 2.0 \mathrm{w} / \mathrm{wt} \%)$

\subsubsection{Cationic polymerisation in EPOX}

Thioxanthone derivatives were also investigated as photosensitizers with an iodonium salt (Iod) for the cationic polymerisation of EPOX upon LED irradiation at $405 \mathrm{~nm}$ to produce either 25 $\mu \mathrm{m}$ thick or $1.4 \mathrm{~mm}$ thick samples under air. For the sake of comparison, polymerisation was also performed with the standard system ITX/Iod. After the irradiation of the system thioxanthone derivative/Iod, a reaction took place as described previously in reaction $\mathrm{r} 3$ and $\mathrm{r} 4$. An electron transfer reaction from the excited thioxanthone derivative to Iod occurs and lead to the formation of an aryl radical $\mathrm{Ar}^{\circ}$ and a radical cation $\mathrm{TX}^{\circ+}$ which can initiate the cationic polymerisation ${ }^{39}$. The polymerisation profiles of EPOX in presence of a photoinitiator/Iod system are presented in the Figure 11. Among the tested thioxanthone derivatives, only ITX allows to obtain a polymer with a tack-free surface. For the polymerisation of $25 \mu \mathrm{m}$ thick sample (Figure 11A), the different systems show an average to low efficiency with a fast kinetic for ITX and slower kinetics for the others compounds. The efficiency trend of thioxanthone derivative as photoinitiator respects the following order: ITX $>$ TX-2DPA $=$ TX-2PTZ $>$ TX2CBZ. The final epoxide function conversions are 59\%, 51\%, 53\% and $22 \%$ respectively. The same polymerisation efficiency and kinetics are observed for $1.4 \mathrm{~mm}$ thick samples (Figure 11B). However, the efficiency trend of thioxanthone derivative as photoinitiator respects the following order: ITX > TX-2CBZ $=\mathrm{TX}-2 \mathrm{DPA}>\mathrm{TX}-2 \mathrm{PTZ}$. The final epoxide function conversions are $51 \%, 35 \%, 34 \%$ and $22 \%$ respectively. The low performance of the new thioxanthone systems could be attributed to the low reactivity of the radical cation toward the monomer.

Unfortunately, among the investigated thioxanthone derivatives for the cationic polymerisation of EPOX, no photoinitiator appears as efficient as ITX. The introduction of amines such as EDB which possess labile hydrogens might improve the polymerisation efficiency by a free radical promoted polymerisation process i.e. the aminoalkyl radicals generated in $\mathrm{r} 2$ can be oxidized to generate additional cations ${ }^{640}$. Another possibility to improve the epoxide function conversion is the synthesis of interpenetrated polymer networks. 
(A)

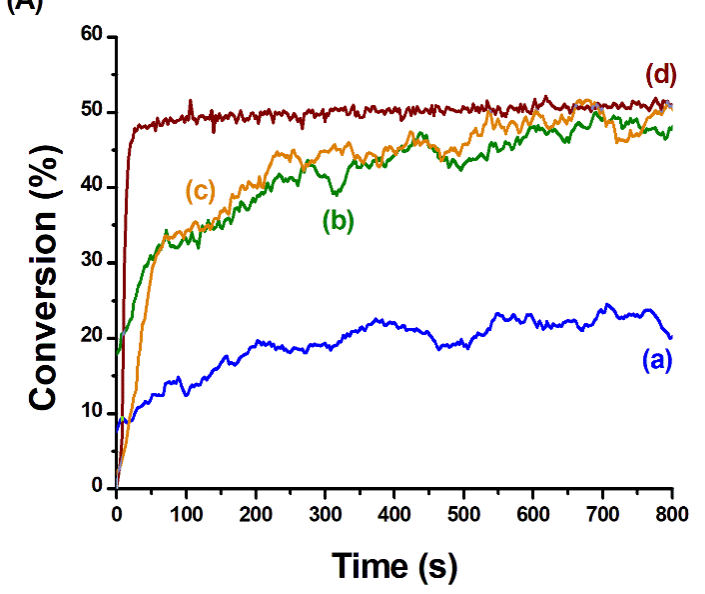

(B)

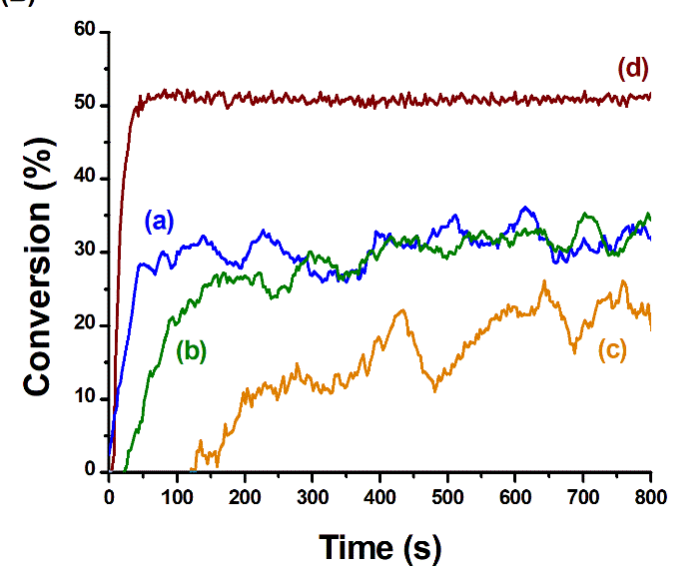

Figure 11: Polymerisation profiles (epoxide function conversion vs irradiation time) of EPOX upon irradiation with a LED at $405 \mathrm{~nm}$, irradiation starts at $10 \mathrm{~s}, 50 \mathrm{~mW} . \mathrm{cm}^{-2}$; (A) sample thickness $=25 \mu \mathrm{m}$ and $(\mathrm{B})$ sample thickness $=1.4 \mathrm{~mm}$. Photoinitiating systems: (curve a) TX2CBZ/Iod (0.07/1.8 w/wt\%), (curve b) TX-2DPA/Iod $(0.22 / 1.9 \mathrm{w} / \mathrm{wt} \%$ ), (curve c) TX2PTZ/Iod $(0.17 / 2.0 \mathrm{w} / \mathrm{wt} \%)$ and (curve d) ITX/Iod $(0.19 / 2.0 \mathrm{w} / \mathrm{wt} \%)$

\subsubsection{IPN synthesis: hybrid polymerisation in TMPTA/EPOX blend}

Thioxanthone derivatives were investigated as photoinitiators with an iodonium salt (Iod) and an amine (EDB) for the polymerisation of a TMPTA/EPOX blend (50/50 w/wt\%) upon LED irradiation at $405 \mathrm{~nm}$ to produce $1.4 \mathrm{~mm}$ thick samples under air (Figure 12). This threecomponent system initiates concomitantly the free radical and the cationic polymerisation to obtain two chemically different interlaced polymer networks which are not covalently bonded. For the sake of comparison and to assess the thioxanthone derivative benefits, polymerisation was also performed with two standard systems i.e. ITX/Iod/EDB and Iod/EDB. Indeed, upon irradiation, iodonium salt Iod and amine EDB could form a charge transfer complex (CTC) which might initiate the polymerisation ${ }^{41}{ }^{42}$. The different systems show a high efficiency toward the free radical polymerisation and an average efficiency toward the cationic polymerisation. Among the tested three-component systems, only those with ITX or TX-2CBZ allow to obtain a polymer with a tack-free surface. The efficiency trend of thioxanthone derivatives in a three-component system respects the following order for both radical and cationic polymerisation: ITX $\approx \mathrm{TX}-2 \mathrm{CBZ}>\mathrm{CTC} \mathrm{Iod} / \mathrm{EDB}=\mathrm{TX}-2 \mathrm{DPA}=\mathrm{TX}-2 \mathrm{PTZ}$. The final $\mathrm{C}=\mathrm{C}$ double bond conversions are $90 \%, 87 \%, 72 \%, 72 \%$ and $72 \%$ respectively. The final epoxide function conversions are $51 \%, 48 \%, 33 \%, 32 \%$ and $22 \%$ respectively. In terms of kinetic, all thioxanthone derivatives induce an increase in the polymerisation speed compared to the control sample containing only Iod and EDB. However, a stronger effect is obtained with ITX and TX-2CBZ for both radical and cationic polymerisations.

The synthesis of interpenetrated polymer networks using a TMPTA/EPOX blend reveals a performance increase for all the investigated systems compared to those used in the free radical polymerisation of TMPTA. In comparison with the two-component systems investigated for the cationic polymerisation of EPOX, only the three-component system, for IPN synthesis, based on TX-2CBZ shows a significant performance increase in both final epoxide function conversion and kinetics. All these improvements can be related to the use of a three-component initiating system. They could also be linked to the synergy between the free radical polymerisation and cationic polymerisation during the IPN synthesis. Indeed, under irradiation, 
while the radical polymerisation process is at first inhibited by the oxygen in the medium, the cationic polymerisation immediately begins which increases the medium viscosity limiting the diffusional oxygen replenishment. The cationic monomer also acts as a diluting agent for the radical polymer network allowing to achieve a higher conversion. Moreover, the exothermicity of the radical polymerisation process tends to boost the cationic polymerisation which is temperature sensitive.

Among the investigated thioxanthone derivatives used for the synthesis of interpenetrated polymer networks in TMPTA/EPOX blend, TX-2CBZ appears as an interesting photoinitiator which exhibits similar performances than ITX but for a lower content due to its better light absorption properties. However, depending on the application in which the polymer is used, other parameters than the photoinitiating system efficiency and kinetics need to be considered such as the polymer colour.

(A)

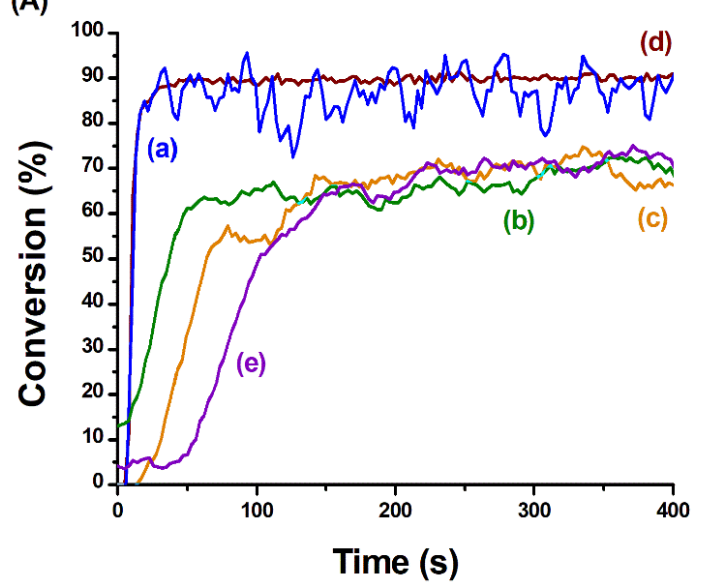

(B)

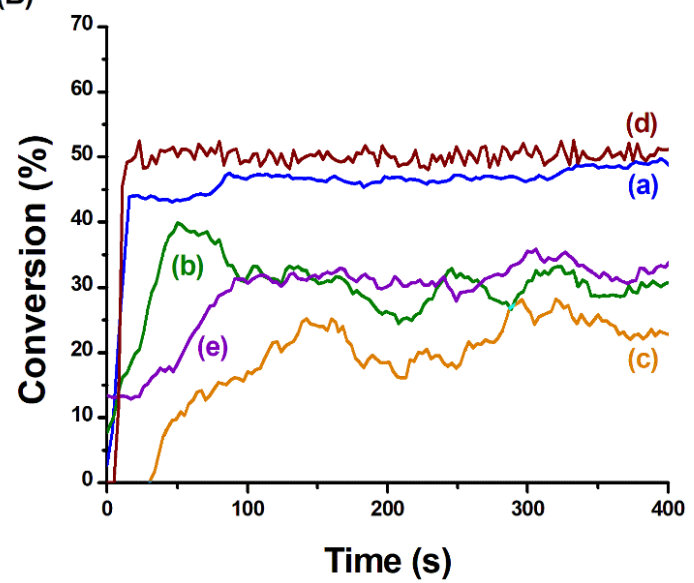

Figure 12: Polymerisation profiles (A) (acrylate function conversion vs irradiation time) and (B) (epoxide function conversion vs irradiation time) of TMPTA/EPOX blend $(50 / 50 \mathrm{w} / \mathrm{wt} \%$ ) upon irradiation with a LED at $405 \mathrm{~nm}$, sample thickness $=1.4 \mathrm{~mm}$, irradiation starts at $10 \mathrm{~s}$, $50 \mathrm{~mW} . \mathrm{cm}^{-2}$. Photoinitiating systems: (curve a) TX-2CBZ/Iod/EDB (0.07/2.0/2.0 w/w/wt\%), (curve b) TX-2DPA/Iod/EDB (0.23/2.0/2.0 w/w/wt\%), (curve c) TX-2PTZ/Iod/EDB $(0.16 / 2.0 / 2.0 \mathrm{w} / \mathrm{w} / \mathrm{wt} \%)$, (curve d) ITX/Iod/EDB $(0.18 / 1.9 / 1.9 \mathrm{w} / \mathrm{w} / \mathrm{wt} \%)$ and (curve e) $\mathrm{Iod} / \mathrm{EDB}(2.0 / 2.0 \mathrm{w} / \mathrm{wt} \%)$

\subsection{Valorisation of the photoinitiating systems containing TX-2CBZ}

To determine to what extent TX-2CBZ could be used in an application as photoinitiator, three current concerns were looked upon: the polymer final colour, the photoinitiator migration after polymerisation and its use in photosensitive resin for laser writing.

\subsubsection{Polymer colorimetric analysis}

To determine to what extent TX-2CBZ could be an alternative to ITX, a colorimetric analysis of the polymerised $1.4 \mathrm{~mm}$ thick samples was performed. The results are presented in Table 2 as CIE L* $a^{*} b^{*}$ parameters. The CIE L* $a^{*} b^{*}$ is a uniform colour space where each colour is represented by three coordinates. $\mathrm{L}^{*}$ characterises the brightness with black and white 
corresponding to value 0 and 100 respectively. $\mathrm{a}^{*}$ indicates the red/green coordinate with positive value for red and negative for green. $b^{*}$ indicates the yellow/blue coordinate with positive value for yellow and negative for blue. Considering the samples prepared with ITX initiating systems as standard, the colour variation generated by the change of photoinitiator could be assessed by a simple subtraction. While using TX-2CBZ in a concentration to have the same optical density at $405 \mathrm{~nm}$ before polymerisation as the standard, no significant colour variation has been detected. Since TX-2CBZ possesses higher molar extinction coefficients in the visible range, the concentration of TX-2CBZ to achieve the same final colour of the polymer is lower which is an advantage for the synthesis of colourless polymers while potentially lowering the safety issue (photoinitiator release).

Table 2: $\mathrm{L}^{*} \mathrm{a}^{*} \mathrm{~b}^{*}$ parameters of $1.4 \mathrm{~mm}$ thick polymer samples obtained thanks to ITX or TX2CBZ initiating systems

\begin{tabular}{lccc}
\hline Initiating system and Monomer & $\mathrm{L}^{*}$ & $\mathrm{a}^{*}$ & $\mathrm{~b}^{*}$ \\
\hline TX-2CBZ / Iod in TMPTA & 52 & 0 & 2 \\
TX-2CBZ / EDB in TMPTA & 54 & 0 & 4 \\
TX-2CBZ / Iod in EPOX & 59 & 0 & 4 \\
TX-2CBZ / Iod / EDB in & 74 & -1 & 8 \\
TMPTA/EPOX & 49 & 0 & 2 \\
ITX / Iod in TMPTA & 54 & -2 & 6 \\
ITX / EDB in TMPTA & 57 & 0 & 4 \\
ITX / Iod in EPOX & 62 & -2 & 8
\end{tabular}

The migration of the photoinitiator after polymerisation is an important topic when selecting a photoinitiator. Indeed, the potential release of photoinitiator may affect the end-use of the polymeric material due to safety issue, in particular for applications related to graphic arts, medicine or food. To assess the migration of the photoinitiator, for either TX-2CBZ or ITX, an experiment with $1.4 \mathrm{~mm}$ thick IPN samples was performed. The polymers were synthesised from a TMPTA-EPOX blend with a three-component photoinitiating system containing either TX-2CBZ or ITX. After an immersion of the sample in acetonitrile for 1h35, the photoinitiator content in the liquid medium was measured by UV-visible absorption spectroscopy and compared with the theoretical amount in the sample. No migration of TX-2CBZ was detected while around $7 \%$ of ITX (molar percentage) could be extracted. Due to the higher molecular 
weight of TX-2CBZ compared to that of ITX (542.65 vs. 254.30 g.mol ${ }^{-1}$ ), these results could be expected. Indeed, higher molar mass tends to limit the diffusion phenomenon in the polymeric material and consequently the migration. Thus, TX-2CBZ, with a similar efficiency as ITX and a lower migration, is an interested photoinitiator and a potential alternative to ITX.

\subsubsection{Photosensitive resin for laser writing experiments}

Laser writing experiment for IPN synthesis using the three-component photoinitiator system, TX-2CBZ/Iod/EDB, in a TMPTA/EPOX blend was performed under air. The sample successfully obtained was analysed by numerical optical microscopy, the 2D and 3D images of the sample are presented in Figure 13. The three-dimensional pattern (TX-2CBZ) was characterized through a profilometric observation to assess the sample thickness $\mathrm{H}$ and the spatial resolution $r$. As expected, efficient photopolymerisation processes occurred in the irradiated area and did not spread out which highlight a good spatial control of the processes. Optical microscopy revealed that the printed sample has a controlled thickness of $1 \mathrm{~mm}(\mathrm{H})$ and a spatial resolution of around $70 \mu \mathrm{m}(\mathrm{r})$ which is slightly higher than the size of the laser beam $(50 \mu \mathrm{m})$. A shrinkage study of the 3D printed sample using Archimede's principle showed a low shrinkage percentage of $1.7 \%$.

(A)

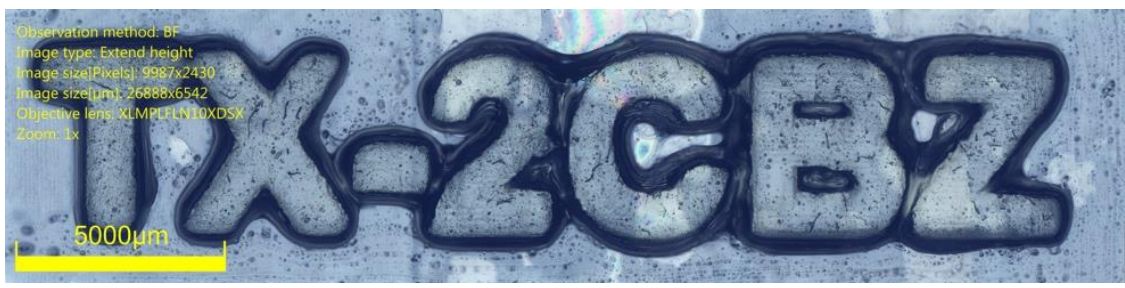

(B)

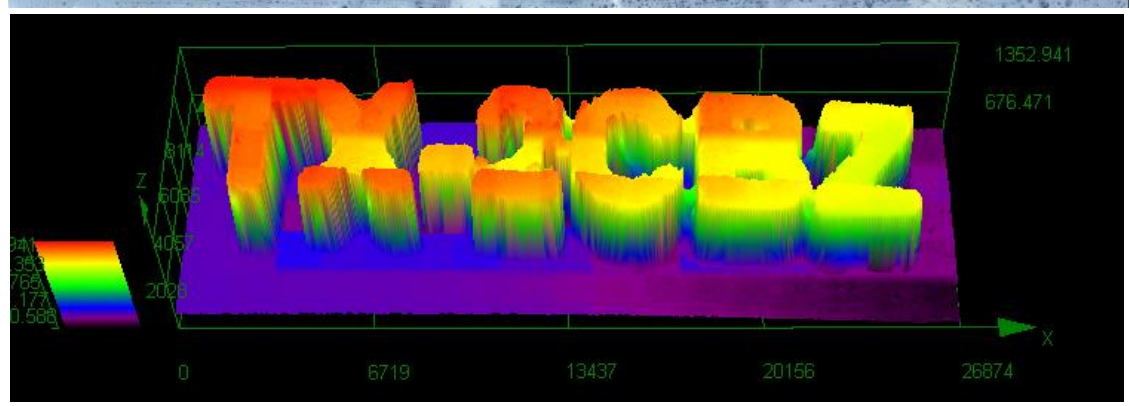

Figure 13 Optical microscopy of the three-dimensional pattern obtained from TX2CBZ/Iod/EDB (0.07/2.0/2.0 w/w/wt $\%)$ in a TMPTA/EPOX blend $(50 / 50 \mathrm{w} / \mathrm{wt} \%)$ (A) $2 \mathrm{D}$ panorama, (B) 3D panorama 


\section{Conclusion}

In the present paper, thioxanthone derivatives are studied in details as potential photoinitiators. The reactivity and efficiency of thioxanthone derivatives have been compared with 2isopropylthioxanthone (ITX) for the free radical polymerisation of TMPTA and the cationic polymerisation of EPOX to produce $25 \mu \mathrm{m}$ and $1.4 \mathrm{~mm}$ thick samples upon LED irradiation at $405 \mathrm{~nm}$. Similarly, their efficiency toward IPN synthesis were evaluated. The reported data reveal an interesting photoinitiator, 2,7-Dicarbazole-9H-thioxanthen-9-one (TX-2CBZ), which tends to have similar or slightly lower performances than the standard photoinitiator (ITX) but with the advantage of a lower content due to better light absorption properties. The chemical mechanisms were characterised thanks to absorption spectroscopy, fluorescence spectroscopy, laser flash photolysis and molecular modelling. Interestingly, TX-2CBZ presents an extended singlet state $\mathrm{S}_{1}$ lifetime compared to thioxanthone which may explain why TX-2CBZ excited state $S_{1}$ is the main photoactivated species involved in the photoinitiating systems. TX-2DPA and TX-2PTZ were not highly reactive and their excited states $S_{1}$ and $T_{1}$ cannot be detected and therefore their $\mathrm{S} 1$ deexcitation are probably governed by internal conversion. The use of a photosensitive resin containing TX-2CBZ for laser writing has been efficiently tested with low shrinkage. TX-2CBZ, with a similar performance for initiating polymerisation as ITX and a lower migration, is an interested photoinitiator. Further work needs to be done to establish whether this photoinitiator is an alternative to ITX in particular in terms of cytotoxicity of the photoinitiating system and the cost of production at an industrial scale.

\section{Acknowledgement}

T.H.L. thanks the Vietnamese government for the doctoral scholarship (program 911 USTH).

The Agence Nationale de la Recherche (ANR agency) is thanked for the funding of the VISICAT project (ANR-17-CE08-0054). 
6121 J. Kirschner, F. Szillat, M. Bouzrati-Zerelli, J.-M. Becht, J. E. Klee and J. Lalevée, Journal of 613 Polymer Science Part A: Polymer Chemistry, , DOI:10.1002/pola.29431.

6142 J. Lalevée, J. P. Fouassier, B. Graff, J. Zhang and P. Xiao, in Photopolymerisation Initiating 615 Systems, 2018, pp. 179-199.

6163 S. C. Ligon, R. Liska, J. Stampfl, M. Gurr and R. Mülhaupt, Chem. Rev., 2017, 117, 1021261710290.

6184 X. Allonas, J. P. Fouassier, M. Kaji, M. Miyasaka and T. Hidaka, Polymer, 2001, 42, 76276197634.

6205 N. Karaca, D. Karaca Balta, N. Ocal and N. Arsu, Journal of Luminescence, 2014, 146, 424621429.

6226 S. Dadashi-Silab, C. Aydogan and Y. Yagci, Polym. Chem., 2015, 6, 6595-6615.

6237 Q. Wu, X. Wang, Y. Xiong, J. Yang and H. Tang, RSC Adv., 2016, 6, 66098-66107.

6248 E. Hola, M. Pilch and J. Ortyl, Catalysts, 2020, 10, 903.

6259 Q. Wu, W. Liao, Y. Xiong, J. Yang, Z. Li and H. Tang, Polymers, 2019, 11, 695.

$62610 \quad$ J. Kreutzer, K. Kaya and Y. Yagci, European Polymer Journal, 2017, 95, 71-81.

62711 S. R. Valandro, A. L. Poli, T. Venâncio, J. Pina, J. S. de Melo, H. D. Burrows and C. C.

628 Schmitt, Journal of Photochemistry and Photobiology A: Chemistry, 2016, 327, 15-20.

62912 T. N. Eren, N. Okte, F. Morlet-Savary, J. P. Fouassier, J. Lalevee and D. Avci, Journal of 630 Polymer Science Part A: Polymer Chemistry, 2016, 54, 3370-3378.

63113 Q. Wu, Y. Xiong, J. Yang, H. Tang and S. Chen, Macromolecular Chemistry and Physics, $632 \quad 2016,217,1569-1578$.

63314 S. Dadashi-Silab, H. Bildirir, R. Dawson, A. Thomas and Y. Yagci, Macromolecules, 2014, $634 \quad 47,4607-4614$.

63515 X. Jiang, J. Luo and J. Yin, Polymer, 2009, 50, 37-41.

63616 B. Gacal, H. Akat, D. K. Balta, N. Arsu and Y. Yagci, Macromolecules, 2008, 41, 2401-2405.

63717 X. Jiang and J. Yin, Macromolecular Chemistry and Physics, 2008, 209, 1593-1600.

63818 Q. Wu, K. Tang, Y. Xiong, X. Wang, J. Yang and H. Tang, Macromolecular Chemistry and 639 Physics, 2017, 218, 1600484.

64019 G. Yilmaz, B. Aydogan, G. Temel, N. Arsu, N. Moszner and Y. Yagci, Macromolecules, $6412010,43,4520-4526$.

64220 F. Karasu, N. Arsu, S. Jockusch and N. J. Turro, Macromolecules, 2009, 42, 7318-7323. 
64522 N. Karaca, D. K. Balta, N. Ocal and N. Arsu, Journal of Polymer Science Part A: Polymer 646 Chemistry, 2016, 54, 1012-1019.

64723 D. K. Balta, G. Temel, G. Goksu, N. Ocal and N. Arsu, Macromolecules, 2012, 45, 119-125.

64824 D. Tunc and Y. Yagci, Polym. Chem., 2011, 2, 2557-2563.

64925 Z. Wang, Y. Li, X. Cai, D. Chen, G. Xie, K. Liu, Y.-C. Wu, C.-C. Lo, A. Lien, Y. Cao and S.650 J. Su, ACS Appl. Mater. Interfaces, 2016, 8, 8627-8636.

65126 M. P. Coleman and M. K. Boyd, J. Org. Chem., 2002, 67, 7641-7648.

65227 T. G. McKenzie, E. H. H. Wong, Q. Fu, A. Sulistio, D. E. Dunstan and G. G. Qiao, ACS 653 Macro Lett., 2015, 4, 1012-1016.

65428 C. Dietlin, S. Schweizer, P. Xiao, J. Zhang, F. Morlet-Savary, B. Graff, J.-P. Fouassier and J. 655 Lalevée, Polym. Chem., 2015, 6, 3895-3912.

65629 J. B. Foresman and Ae. Frisch, .

$65730 \quad$ J. Lee, K. Shizu, H. Tanaka, H. Nakanotani, T. Yasuda, H. Kaji and C. Adachi, J. Mater. 658 Chem. C, 2015, 3, 2175-2181.

65931 J. Christmann, X. Allonas, C. Ley, A. Ibrahim and C. Croutxé-Barghorn, Macromolecular 660 Chemistry and Physics, 2017, 218, 1600597.

66132 S. L. Murov, I. Carmichael and G. L. Hug, Handbook of Photochemistry, Second Edition, 662 CRC Press, 2nd ed., rev.expanded., 1993.

66333 M. G. Neumann, M. H. Gehlen, M. V. Encinas, N. S. Allen, T. Corrales, C. Peinado and F. 664 Catalina, J. Chem. Soc., Faraday Trans., 1997, 93, 1517-1521.

$66534 \quad$ S. F. Yates and G. B. Schuster, J. Org. Chem., 1984, 49, 3349-3356.

66635 N. J. Turro, V. Ramamurthy and J. C. Scaiano, Modern Molecular Photochemistry of Organic 667 Molecules, University Science Books, 2010.

$66836 \quad$ W. H. Melhuish, J. Phys. Chem., 1961, 65, 229-235.

66937 J. Lalevée, M. El-Roz, X. Allonas and J. P. Fouassier, Journal of Polymer Science Part A: 670 Polymer Chemistry, 2008, 46, 2008-2014.

67138 D. G. Anderson, R. Stephen Davidson and J. J. Elvery, Polymer, 1996, 37, 2477-2484.

67239 W. D. Cook, S. Chen, F. Chen, M. U. Kahveci and Y. Yagci, Journal of Polymer Science Part 673 A: Polymer Chemistry, 2009, 47, 5474-5487.

67440 M. A. Tasdelen, J. Lalevée and Y. Yagci, Polym. Chem., 2020, 11, 1111-1121.

67541 P. Garra, B. Graff, F. Morlet-Savary, C. Dietlin, J.-M. Becht, J.-P. Fouassier and J. Lalevée, 676 Macromolecules, 2018, 51, 57-70. 
67742 D. Wang, A. Arar, P. Garra, B. Graff and J. Lalevée, Journal of Polymer Science, 2020, 58, $678 \quad 811-823$.

679 
682

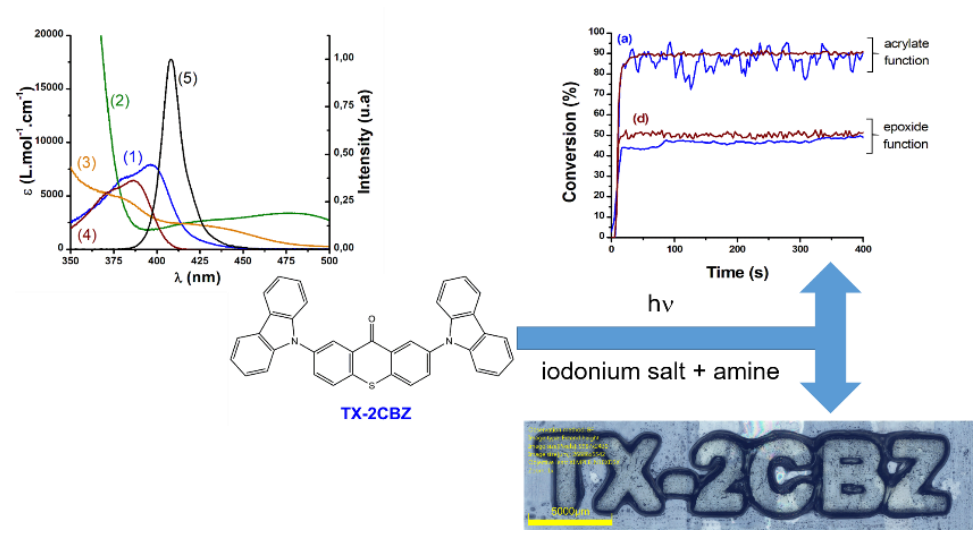

